\title{
Identification of Ftr1 and Zrt1 as iron and zinc micronutrient transceptors for activation of the PKA pathway in Saccharomyces cerevisiae
}

\author{
Joep Schothorst ${ }^{1,2}$, Griet Van Zeebroeck ${ }^{1,2}$ and Johan M. Thevelein ${ }^{1,2, *}$ \\ ${ }^{1}$ Laboratory of Molecular Cell Biology, Institute of Botany and Microbiology, KU Leuven, Belgium. \\ ${ }^{2}$ Department of Molecular Microbiology, VIB, Kasteelpark Arenberg 31, B-3001 Leuven-Heverlee, Flanders, Belgium. \\ * Corresponding Author: \\ Johan M. Thevelein, Tel: +32 16 321507; Fax: +32 16 321979; E-mail: johan.thevelein@mmbio.vib-kuleuven.be
}

\begin{abstract}
Multiple types of nutrient transceptors, membrane proteins that combine a transporter and receptor function, have now been established in a variety of organisms. However, so far all established transceptors utilize one of the macronutrients, glucose, amino acids, ammonium, nitrate, phosphate or sulfate, as substrate. This is also true for the Saccharomyces cerevisiae transceptors mediating activation of the PKA pathway upon re-addition of a macronutrient to glucose-repressed cells starved for that nutrient, reestablishing a fermentable growth medium. We now show that the yeast high-affinity iron transporter Ftr1 and high-affinity zinc transporter Zrt1 function as transceptors for the micronutrients iron and zinc. We show that replenishment of iron to iron-starved cells or zinc to zinc-starved cells triggers within 1-2 minutes a rapid surge in trehalase activity, a well-established PKA target. The activation with iron is dependent on Ftr1 and with zinc on Zrt1, and we show that it is independent of intracellular iron and zinc levels. Similar to the transceptors for macronutrients, Ftr1 and Zrt1 are strongly induced upon iron and zinc starvation, respectively, and they are rapidly downregulated by substrate-induced endocytosis. Our results suggest that transceptormediated signaling to the PKA pathway may occur in all cases where glucoserepressed yeast cells have been starved first for an essential nutrient, causing arrest of growth and low activity of the PKA pathway, and subsequently replenished with the lacking nutrient to re-establish a fermentable growth medium. The broadness of the phenomenon also makes it likely that nutrient transceptors use a common mechanism for signaling to the PKA pathway.
\end{abstract}

\author{
doi: 10.15698/mic2017.03.561 \\ Received originally: 05.11.2016; \\ in revised form: 28.01.2017, \\ Accepted 31.01.2017, \\ Published 02.03.2017.
}

Keywords: Ftr1, Zrt1, iron, zinc, transceptor, signaling, PKA.

\section{INTRODUCTION}

As a unicellular eukaryotic micro-organism, the yeast Saccharomyces cerevisiae has to be able to rapidly adapt to severe fluctuations in the extracellular conditions. One of the main fluctuating parameters in the extracellular environment is nutrient availability. Hence, microorganisms like yeast have developed a multitude of complex nutrientsensing mechanisms in order to properly respond to fluctuations in the supply of specific nutrients and to adapt cellular metabolism, growth and development accordingly. Among eukaryotic microorganisms, these mechanisms have by far been elucidated in greatest detail in $S$. cerevisiae $[1,2]$. One of the most common adaptation mechanisms with respect to nutrient availability is the induction of high-affinity transporters for specific essential nutrients lacking or limiting in the medium, so that trace amounts of the sparse nutrient can be taken up efficiently by the microorganism. Examples of this adaptation mechanism have been described in yeast for all essential nutrients, i.e. the macronutrients glucose, nitrogen, phosphate and sulfate, and micronutrients like metal ions and vitamins. Limitation for glucose causes upregulation of Hxt6 and Hxt7 [3], for nitrogen the general amino acid permease Gap1 [4] and ammonium transporters Mep1 and Mep 2 [5], for phosphate Pho84 [6], for sulfate Sul1 and Sul2 [7], for iron Ftr1 [8] and for Zinc Zrt1 [9]. Even for non-essential nutrients like uracil, uracil permease is induced in media lacking uracil [10]. The high-affinity transporters generally undergo rapid downregulation upon re-exposure to their substrate through endocytic internalization, which is induced by 
ubiquitination, followed by sorting to the multivesicular body (MVB) and subsequently by vacuolar degradation [1014].

Next to the upregulation of high-affinity transporters in response to starvation for specific nutrients, the yeast $S$. cerevisiae has a multitude of different nutrient-sensing pathways at its disposal [1, 2]. One of these nutrientsensing pathways is the Fermentable Growth Medium (FGM) induced pathway, which maintains high activity of PKA in a medium with a fermentable sugar and all other nutrients required for growth. Deprivation for any of the other essential nutrients in the presence of a fermentable sugar causes growth arrest and downregulation of PKA activity, while subsequent re-addition of the missing nutrient triggers rapid modulation of PKA targets, such as activation of the enzyme trehalase by phosphorylation, during the resumption of fermentable growth $[15,16]$. This nutrient activation of the PKA pathway is not triggered by an increase in CAMP as in the case of glucose activation of the PKA pathway in glucose-derepressed yeast cells [16]. The signaling mechanism remains elusive.

We have shown that activation of the PKA pathway targets by the other nutrients besides glucose, is mediated by high-affinity transporters induced during the starvation period that function as transporter-receptors or 'transceptors': Gap1 for amino acids [17, 18], Pho84 for phosphate [19-21], Mep2 for ammonium [5] and Sul1,2 for sulfate [22]. Major arguments include the activation by nontransported and/or non-metabolized analogs and the separation of transport and signaling by specific mutations. For each of these transceptors, starvation for their substrate triggers strong upregulation at the transcriptional level, while re-addition of the missing substrate triggers rapid endocytosis and vacuolar degradation. As these proteins combine a transporter function with a receptor function, we have named them transceptors [23]. We have proposed that a specific conformation has to be induced in the transceptor to initiate signaling, which appears to be dependent on the structure of the substrate, since not all transported substrates are able to trigger signaling $[18,24]$ Transceptors may constitute an intermediate step in the development of receptors from transporters during evolution [25].

After the initial identification of nutrient transceptors in S. cerevisiae, the concept of transceptors has spread to other organisms, ranging from yeasts and fungi to higher eukaryotic organisms including plants, mice and humans [26]. However, while an increasing number of transceptors in multiple organisms has now been identified, their substrates, glucose, phosphate, amino acids, ammonium, nitrate and sulfate, all belong to the class of macronutrients. Up to now, no transceptors for micronutrients, like metal ions or vitamins, have been identified. Since starvation for an essential micronutrient also causes growth arrest and entry into stationary phase, it is expected that the PKA pathway will also be downregulated and that re-addition of the lacking micronutrient may cause a similar sudden activation of the PKA pathway as is observed after starvation and re-addition of a macronutrient. This is especially true since high-affinity transporters for micronutrients, like metal ions, are well known to be strongly induced also by starvation for their substrate and rapidly downregulated by endocytosis and breakdown in the vacuole after readdition of the lacking micronutrient $[8,9,14,27]$. Based on these criteria we selected two known cation transporters, the high-affinity iron transporter Ftr1 and the highaffinity zinc transporter Zrt1, as potential micronutrient transceptors.

Ftr1 is a high-affinity $\mathrm{Fe}^{3+}$ transporter, which together with the Fet3 oxidoreductase forms the high-affinity iron uptake system. Uptake of $\mathrm{Fe}^{2+}$ by Ftr1 is preceded by oxidation of $\mathrm{Fe}^{2+}$ to $\mathrm{Fe}^{3+}$, followed by inter-complex channelling of $\mathrm{Fe}^{3+}$ to Ftr1 and subsequent transport over the plasma membrane $[8,28-30]$. The strong interplay between Ftr1 and Fet 3 is also shown by the fact that they need each other for proper targeting to the plasma membrane [8]. As mentioned above, FTR1 expression is strongly upregulated under iron limitation [8], which is controlled mainly by the two transcription factors, Aft1 and Aft2 [31, 32]. Upon iron limitation, Aft1 accumulates in the nucleus and induces the expression of FTR1 and FET3 [33]. Ftr1 is also subjected to regulation at the post-translational level. When iron is again available, Ftr1 is rapidly endocytosed and degraded presumably to prevent overaccumulation of iron to toxic levels [14]. Ftr1 endocytosis is mediated by ubiquitination and requires an active Ftr1-Fet3 complex. Hence, Ftr1 shows a very similar expression pattern as the macronutrient transceptors, being present at the plasma membrane under substrate limiting conditions and being rapidly removed from the plasma membrane when the substrate becomes plentiful again.

Upon zinc limitation, S. cerevisiae stimulates zinc uptake to ensure zinc homeostasis. S. cerevisiae has three different zinc uptake systems: a high-affinity zinc transporter $\left(\mathrm{K}_{\mathrm{m}} \approx 1 \mu \mathrm{M}\right)$ Zrt1 [9], a low-affinity zinc transporter $\left(K_{\mathrm{m}} \approx 10 \mu \mathrm{M}\right)$ Zrt2 [34] and the non-specific Fet4 transporter [35]. Expression of both ZRT1 and ZRT2 is regulated through the activity of the Zap1 transcription factor [36]. The expression of ZAP1 is strongly stimulated in response to zinc limitation and its enhanced activity strongly induces expression of ZRT1 while inhibiting expression of ZRT2 [31, $37,38]$. In analogy with Ftr1 and the known macronutrient transceptors, the high-affinity zinc transporter Zrt1 is also rapidly removed from the plasma membrane upon exposure to high extracellular zinc concentrations [9]. This process is mediated by endocytosis following ubiquitination and subsequent vacuolar degradation and can be induced by high levels of cadmium and cobalt as well [27, 39].

We now show that both iron and zinc limitation lead to downregulation of PKA pathway targets during growth arrest and that re-addition of iron or zinc, respectively, triggers the same rapid activation of the PKA target trehalase as previously observed with the macronutrients. We provide evidence that signaling is independent of the intracellular iron or zinc levels, and that the high-affinity transporters, Ftr1 and Zrt1, have an additional sensing function for activation of the PKA pathway, next to their transport function. Hence, we have identified Ftr1 and Zrt1 as the 
first transceptors for micronutrients, which significantly broadens the concept of nutrient transceptors.

\section{RESULTS}

Influence of iron and zinc deprivation on PKA pathway targets and expression of Ftr1 and Zrt1

We have tested the effect of different iron and zinc deprivation conditions on arrest of cell proliferation and a set of well-established read-outs for activity of the PKA pathway, in particular the accumulation of the storage carbohydrates trehalose and glycogen. Cell proliferation was rapidly inhibited upon imposing starvation for iron using an iron deprivation medium supplemented with iron specific che- lators in order to neutralize minute amounts of iron present in the milliQ water used to prepare the medium (Fig. $1 \mathrm{~A})$. We have used the membrane impermeable chelator bathophenantroline disulfonate (BPS) and the membrane permeable chelator ferrozine/3-(2-Pyridyl)-5,6-diphenyl1,2,4-triazine- $p, p^{\prime}$-disulfonic acid monosodium salt hydrate) for iron deprivation. For zinc starvation we have used a medium deprived of zinc and supplemented with the non-specific ethylenediaminetetraacetic acid (EDTA) chelator. In all conditions tested, growth was virtually inhibited within 24 to $30 \mathrm{~h}$ after the onset of metal ion deprivation (Fig. 1A). Consistently, the percentage of unbudded cells had significantly increased after $24 \mathrm{~h}$ of metal ion
A

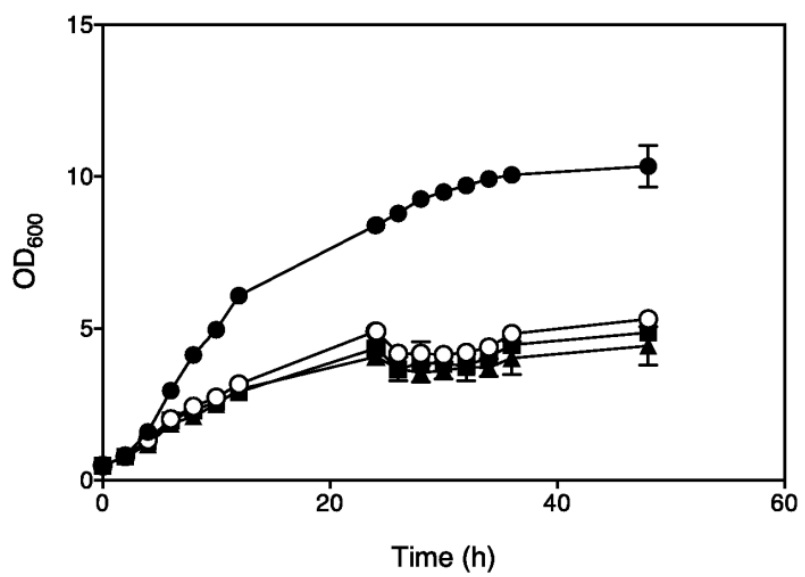

C

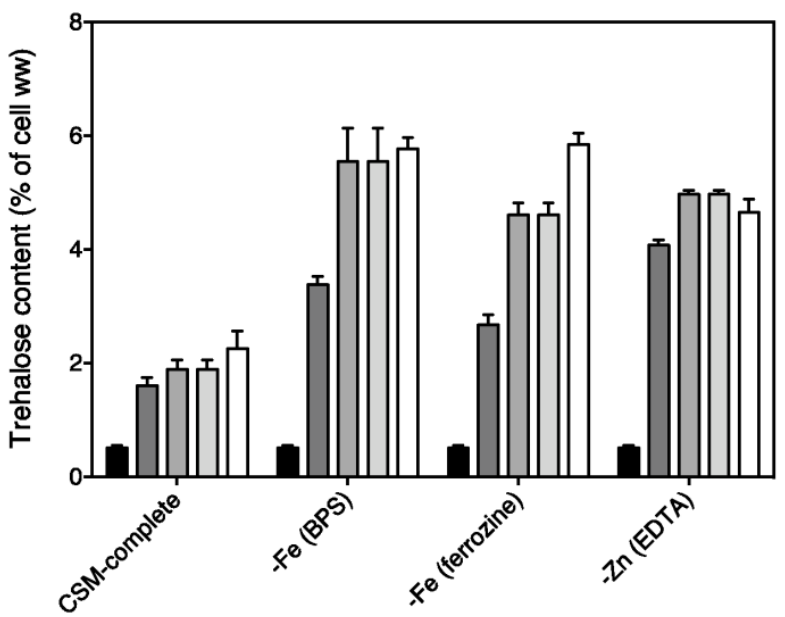

B

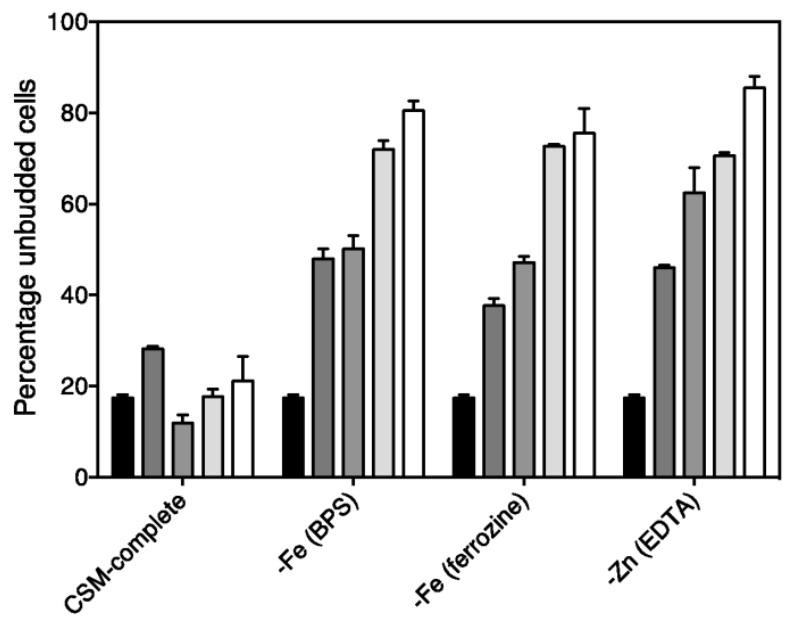

D

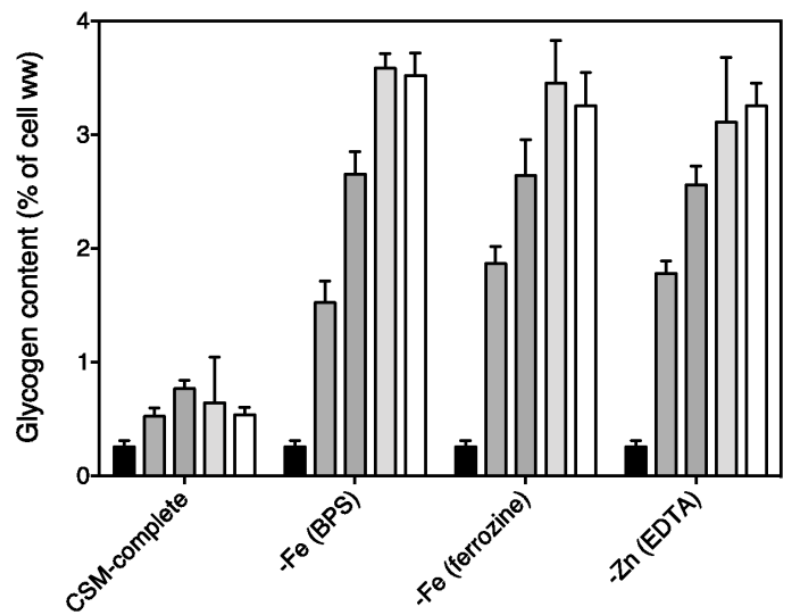

FIGURE 1: Growth rate, budding percentage, and trehalose and glycogen accumulation upon deprivation for iron or zinc. All experiments were performed with the $\Sigma 1278$ wild type strain. All samples originate from a preculture that was grown overnight in all trace medium, and subsequently diluted at $\mathrm{t}=0$ to an $\mathrm{OD}_{600}$ of 0.5 in the indicated deprivation media. (A) Growth inhibition upon exposure to different iron or zinc deprivation conditions, $\mathrm{n}=2$. Cells were put on fresh deprivation medium each $24 \mathrm{~h}$ and $\mathrm{OD}_{600} \mathrm{was}_{\mathrm{s}}$ measured at regular intervals. (closed circles) all trace medium, (open circles) iron deprivation with BPS, (closed squares) iron deprivation with ferrozine, (closed triangles) zinc deprivation with EDTA. (B) Percentage of unbudded cells in response to the indicated deprivation conditions with CSM complete as control condition, $n=2$. (C) Accumulation of trehalose in response to the indicated deprivation conditions, $n=2$. (D) Accumulation of glycogen in response to the indicated deprivation conditions, $n=2$. (B,C,D) The shaded bars indicate non-starved cells and $1,2,3$ or 4 days of metal ion starvation. 
starvation and reached about $80 \%$ after $72 \mathrm{~h}$ of starvation (Fig. 1B). The trehalose content rapidly increased under all metal ion deprivation conditions to reach a maximum approximately after $48 \mathrm{~h}$ (Fig. 1C) while glycogen content increased more slowly to reach a maximum after about 72 h (Fig. 1D). This indicates that entrance of stationary phase caused by iron or zinc deprivation is also associated with development of a low-PKA phenotype.
Using qPCR on RNA samples extracted from the cells, we show that under the appropriate metal ion deprivation conditions the expression of FTR1 and ZRT1 is strongly induced, with FTR1 expression continuing to increase during the 4-day iron starvation period (Fig. 2A), while ZRT1 expression reached a maximum already after $48 \mathrm{~h}$ of zinc starvation (Fig. 2B). FTR1 expression was not upregulated under zinc deprivation conditions, while ZRT1 expression
A

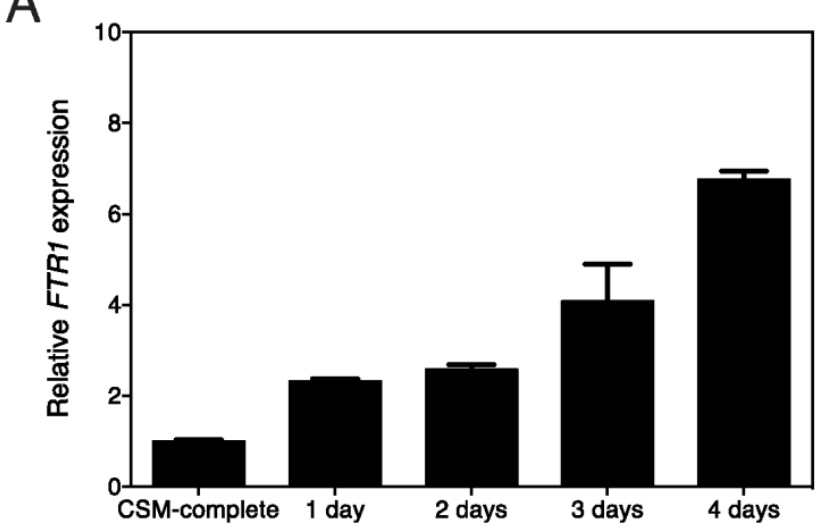

C

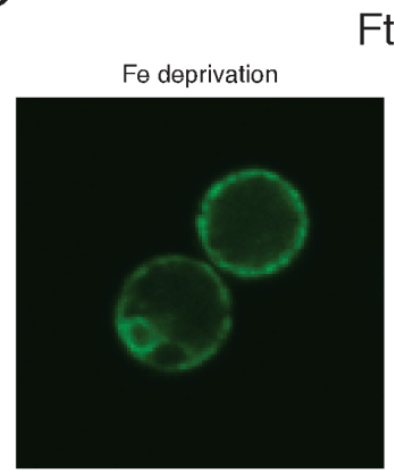

Ftr1

E

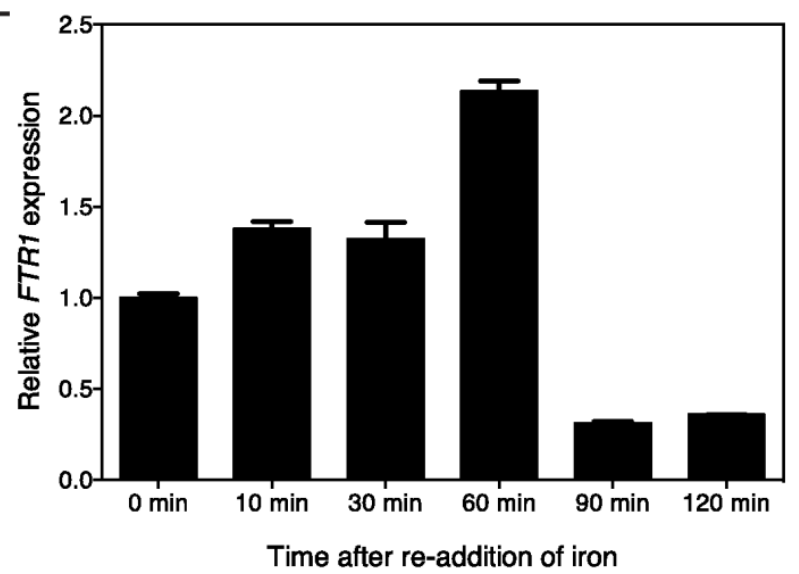

$B$

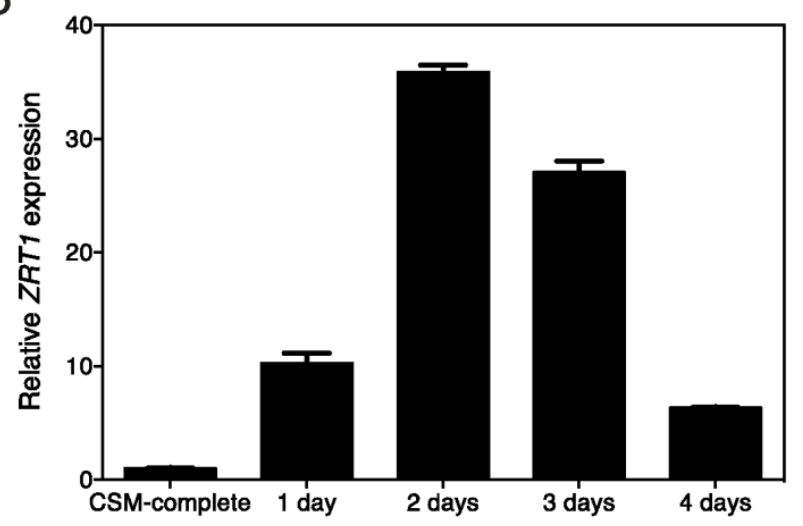

D
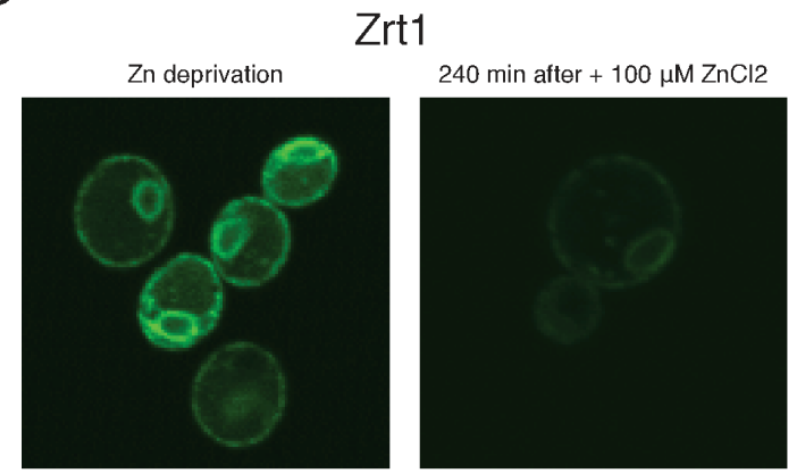

$\mathrm{F}$

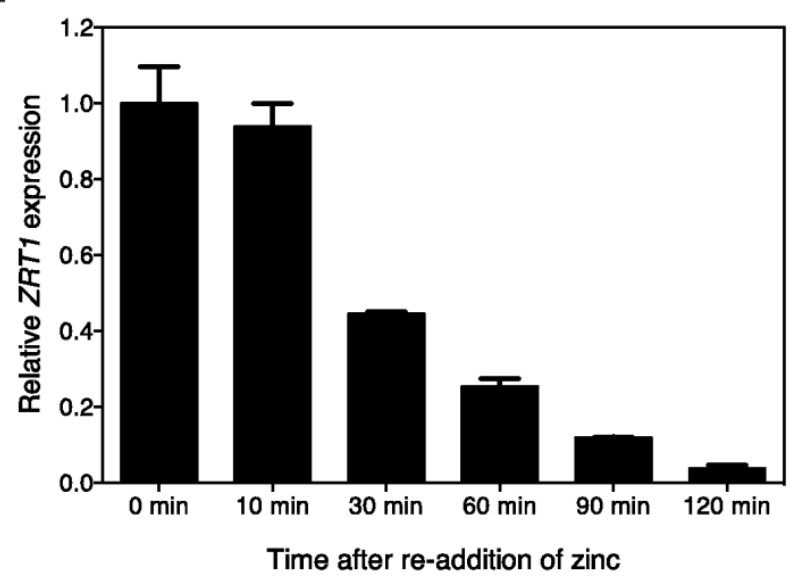

FIGURE 2: Expression level of FTR1 and ZRT1 and cellular localization of Ftr1 and Zrt1 upon iron or zinc deprivation and re-addition. Relative expression of FTR1 (A) and ZRT1 (B) in response to deprivation of iron or zinc, respectively, $\mathrm{n}=2$. As listed in Materials \& Methods, a specific set of reference genes was used depending on the experiment. Localization of C-terminally tagged Ftr1-GFP (C) and Zrt1-GFP (D) under their substrate deprivation conditions (left panel) as well as after re-addition of $100 \mu \mathrm{M}$ of the missing metal ion for 240 min (right panel), $\mathrm{n}=2$. Relative expression of FTR1 (E) and ZRT1 (F) at different time points after re-addition of $100 \mu \mathrm{M}$ of the missing metal ion, iron or zinc, respectively, $\mathrm{n}=2$. 
was not upregulated under iron deprivation conditions supporting the specificity of the starvation conditions. We also determined the cellular localization of Ftr1 and Zrt1 using genomically GFP-tagged constructs. This showed that under iron or zinc deprivation conditions Ftr1 or Zrt1, respectively, accumulated at the plasma membrane and to a smaller extent at an intracellular membrane, possibly the ER membrane since the GFP tag might delay ER exit (Fig. 2C, D). Addition of $100 \mu \mathrm{M}$ iron or zinc for 240 min caused virtual disappearance at the plasma membrane of the Ftr1 or Zrt1 signal, respectively (Fig. 2C, D). Transcriptional expression of FTR1 and ZRT1 was also downregulated after re- addition of $100 \mu \mathrm{M}$ iron or zinc, respectively (Fig. 2E, F).

The phenotype caused by iron or zinc deficiency is specific since it is reversed by re-addition of only iron or zinc We have checked the specificity of the iron and zinc starvation regime by testing the reversibility of the effect on growth, budding percentage and targets of the PKA pathway. This was especially important for zinc since we added the unspecific chelator EDTA to the zinc deprivation medium to ensure thorough zinc starvation. Re-addition of only iron or only zinc to cells starved for four days for either iron or zinc, respectively, caused in each case rapid recovery of
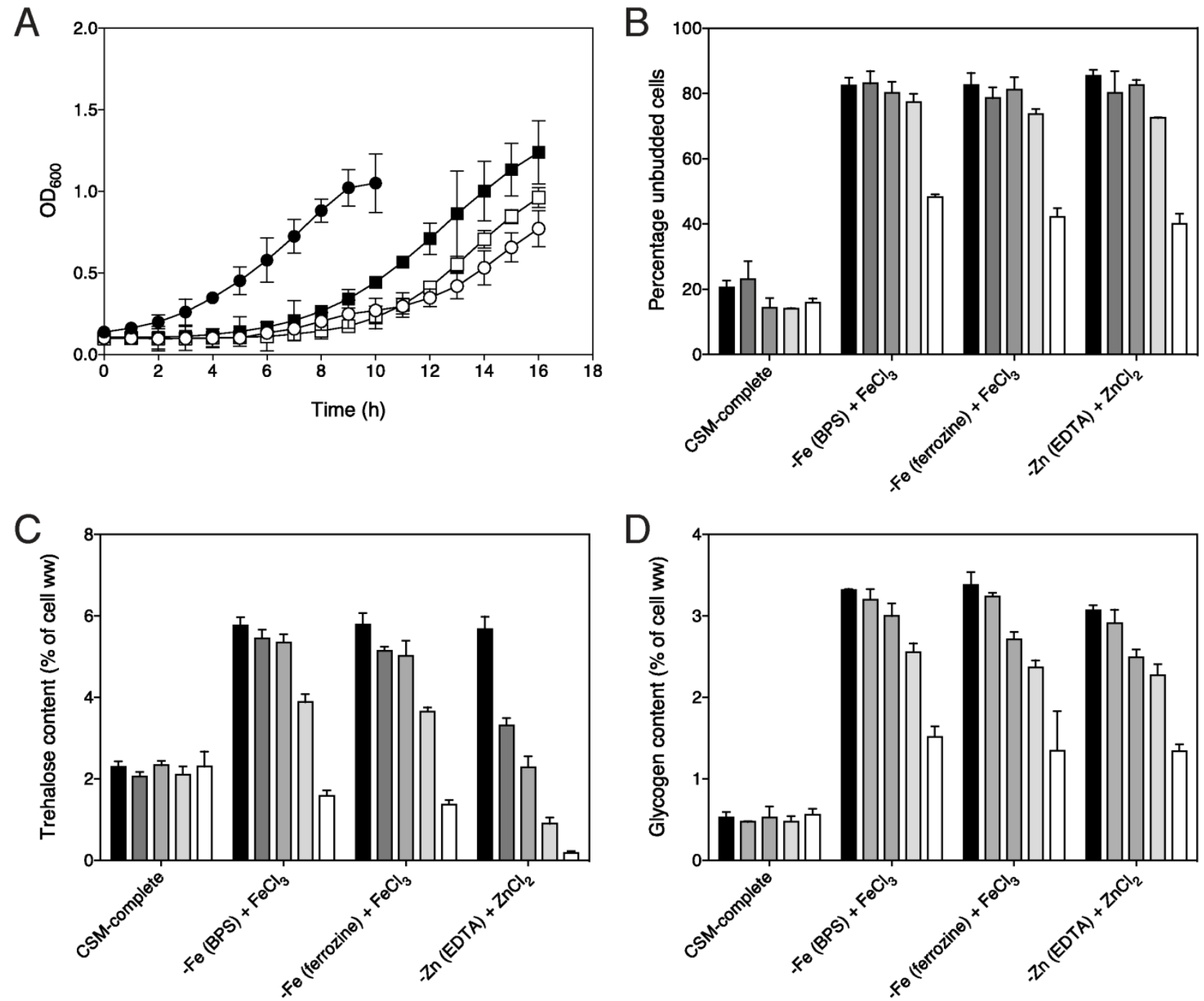

FIGURE 3: Reversal of the low-PKA phenotype developed in response to iron or zinc deprivation is specific for iron or zinc, respectively. (A) Growth induction by re-addition of $100 \mu \mathrm{M}$ of either $\mathrm{FeCl}_{3}$ or $\mathrm{ZnCl}_{2}$ at $\mathrm{t}=0$ to cells that had been deprived for 4 days in the respective metal ion deprivation media. Growth was measured by determination of $\mathrm{OD}_{600}$. (closed circles) all trace medium, (closed squares) ferrozine iron starvation medium $+\mathrm{FeCl}_{3}$, (open squares) EDTA zinc starvation medium $+\mathrm{ZnCl}_{2}$, (open circles) BPS iron starvation medium $+\mathrm{FeCl}_{3}, \mathrm{n}=2$ (B) Percentage of unbudded cells after re-addition of $100 \mu \mathrm{M}$ of either $\mathrm{FeCl}_{3}$ or $\mathrm{ZnCl}_{2}$ at $\mathrm{t}=0$ to cells that had been deprived for 4 days in the respective metal ion deprivation media indicated. The shaded bars represent $0,2,4,8$ and $12 \mathrm{~h}$ after metal ion re-addition, $\mathrm{n}=2$. (C) Trehalose mobilization after re-addition of $100 \mu \mathrm{M}$ of either $\mathrm{FeCl}_{3}$ or $\mathrm{ZnCl}_{2}$ at $\mathrm{t}=0$ to cells that had been deprived for 4 days in the respective metal ion deprivation media indicated or had not been starved for iron (CSM-complete). (D) Glycogen mobilization after re-addition of $100 \mu \mathrm{M}$ of either $\mathrm{FeCl}_{3}$ or $\mathrm{ZnCl}_{2}$ at $\mathrm{t}=0$ to cells that had been deprived for 4 days in the respective metal ion deprivation media indicated or had not been starved for iron (CSM-complete). (C,D) The shaded bars represent $0,30,60,120$ and 240 min after metal ion re-addition, $n=2$. 
growth (Fig. 3A), indicating that the cells were specifically arrested because of lack of either iron or zinc. Iron-induced growth recovery after BPS-elicited iron deprivation was slightly slower than after ferrozine-elicited starvation, suggesting that BPS may cause more stringent iron deprivation or may reduce to some extent the level of one or more other metal ions. The resumption of growth was correlated with a proportionate reduction in the number of unbudded cells (Fig. 3B). The storage carbohydrates trehalose and glycogen were mobilized within the first four $h$ after readdition of the metal ion with the drop in trehalose content being more pronounced than that in glycogen (Fig. 3C, D). Mobilization of trehalose and glycogen preceded significant recovery of growth. In contrast, addition of the same concentration of iron or zinc to non-starved cells did not result in any mobilization of trehalose or glycogen (Fig. 3C, D: CSM-complete). These results show that both growth resumption and downregulation of PKA pathway targets can be induced by re-addition of only iron to iron-starved cells and only zinc to zinc-starved cells, supporting the notion that the cells were truly starved for iron and zinc, respectively. Furthermore, for our purposes of studying metal ion induced activation of the PKA pathway, a two-day starvation period appeared to be appropriate. Iron deprivation with the BPS chelator appeared to be more reproducible than with the ferrozine chelator and was thus used in the following experiments.

Re-addition of iron to iron-deprived cells triggers a rapid, transient Ftr1-dependent activation of trehalase

After establishing that iron and zinc deprivation resulted in a low-PKA phenotype as well as the induction and localization of Ftr1 and Zrt1 at the plasma membrane, we tested the influence of iron and zinc re-addition on short-term activation of the PKA pathway. We used the wellestablished downstream target trehalase as a direct readout for PKA activity $[15,22]$. This showed that very low iron concentrations, as low as $100 \mathrm{nM}$, in the form of $\mathrm{FeCl}_{3}$ or $\mathrm{FeCl}_{2}$ were able to trigger a rapid, transient increase in trehalase activity in cells starved for two days for iron using the BPS chelator (Fig. 4A, B). Concentrations higher than 1 $\mu \mathrm{M} \mathrm{FeCl}{ }_{3}$ or $\mathrm{FeCl}_{2}$ did not enhance trehalase activation further. Subsequently, we investigated whether this trehalase activation was dependent on the iron transporter, Ftr1. Deletion of FTR1, or deletion of FET3, which results in mislocalization of Ftr1 [8], completely abolished iron-induced trehalase activation both with 1 and $100 \mu \mathrm{M} \mathrm{FeCl}_{3}$ (Fig. 4C) or $\mathrm{FeCl}_{2}$ (Fig. 4D), indicating that iron-induced signaling to the PKA pathway is dependent on Ftr1.

\section{Iron-induced signaling does not depend on intracellular iron entry}

Next, we investigated whether Ftr1-dependent ironinduced signaling originates from the plasma membrane, or requires iron uptake in the cytoplasm triggering an intracellular iron response. The first case would suggest that Ftr1 acts as an iron transceptor while the second case would indicate an unknown intracellular iron sensing mechanism. To distinguish between the two possibilities, we measured $\mathrm{Fe}^{55}$ uptake upon re-addition of different iron concentrations to iron-starved cells and compared the uptake in the wild type with that in the $f \operatorname{tr} 1 \Delta$ strain. At the high concentration of $100 \mu \mathrm{M}$, iron uptake in the ftr1 $\Delta$ strain was hardly impaired compared to that in the wild type strain, while at concentrations of $10 \mu \mathrm{M}$ and lower iron uptake was strongly reduced in the $f t r 1 \Delta$ strain (Fig. $4 \mathrm{E}$ ). High concentrations of iron are apparently taken up also by other carriers. Since iron-induced trehalase activation was completely abolished in the $f \operatorname{tr} 1 \Delta$-strain at a concentration of $100 \mu \mathrm{M}$, we can conclude that iron entry into the cytosol is not sufficient to trigger trehalase activation and that therefore the signaling must originate from the Ftr1 carrier in the plasma membrane which apparently plays an essential role as transceptor. We also tested which other transporters could be involved in the uptake of high concentrations of iron using a series of carrier deletion strains. The smf $2 \Delta$ strain showed the largest reduction in uptake of $100 \mu \mathrm{M}$ iron suggesting that Smf2 is the main alternative transporter for iron uptake under these conditions (Fig. 4F).

\section{Re-addition of zinc to zinc-starved cells triggers a rapid,} transient Zrt1-dependent activation of trehalase

Re-addition of zinc to zinc-deprived cells triggered a very similar rapid and transient increase in trehalase activity as in the case of iron re-addition to iron-starved cells (Fig. 5A). The activation was concentration-dependent, with $100 \mathrm{nM}$ of $\mathrm{ZnCl}_{2}$ barely able to induce an increase in trehalase activity, $500 \mathrm{nM} \mathrm{ZnCl}_{2}$ caused an intermediate level of activation, while concentrations above $1 \mu \mathrm{M} \mathrm{ZnCl}_{2}$ triggered maximal trehalase activation. Next, we tested the importance of Zrt1 for zinc-induced trehalase activation. Deletion of ZRT1 resulted in a severe reduction of zinc-induced trehalase activation. Even with the higher concentrations, 10, 100 and $1000 \mu \mathrm{M} \mathrm{ZnCl}_{2}$, which fully activate trehalase in the wild-type strain, trehalase activation was almost absent in the zrt1 $\Delta$ strain (Fig. 5B). As opposed to the complete absence of iron-induced trehalase activation in the ftr1 $\Delta$ strain, some very limited residual zinc-induced trehalase activation was present in the $z r t 1 \Delta$ strain. These results indicate that zinc-induced signaling to the PKA pathway is largely dependent on the Zrt1 transporter, with some unknown transporter(s) being able to sustain slight residual signaling. We also demonstrate that zinc-induced trehalase activation is correlated with phosphorylation of trehalase on Ser21 and Ser83 using phospho-specific antibodies (Fig. 5C), as previously shown for glucose, nitrogenand sulfate-induced trehalase activation in appropriatelystarved cells $[15,22]$. The transient increase in trehalase activity does not precisely correlate with the extent of phosphorylation. This can be explained by the fact that phosphorylation is not the only determinant of trehalase enzymatic activity $[15,22]$. Phosphorylation is also required for binding of the 14-3-3 proteins, which strongly enhance trehalase enzymatic activity, and the Dcs1 inhibitor prevents binding of the $14-3-3$ proteins (Bmh1/2) to trehalase. Hence, phosphorylation can still be high while the activity may already be reduced. 
A

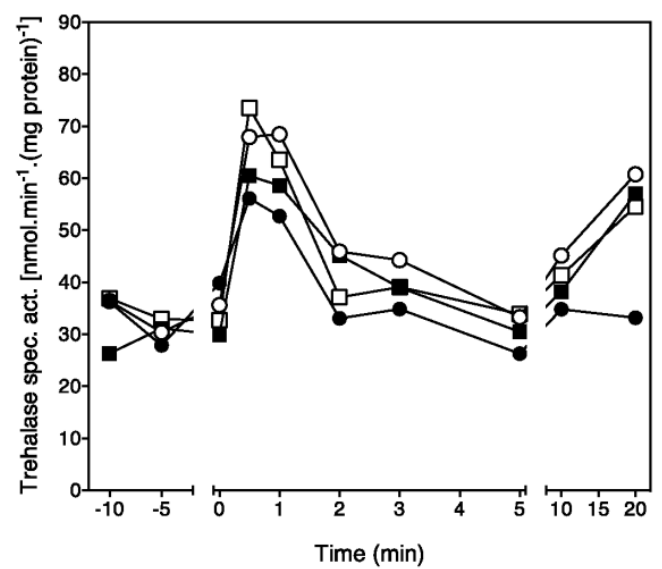

C

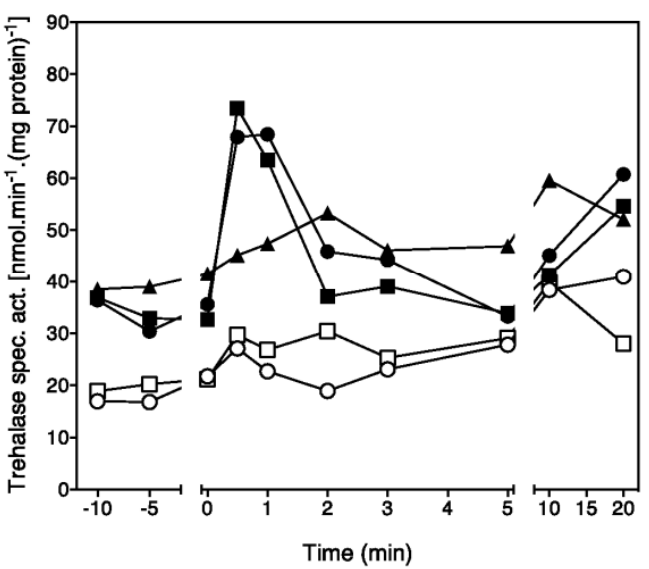

E

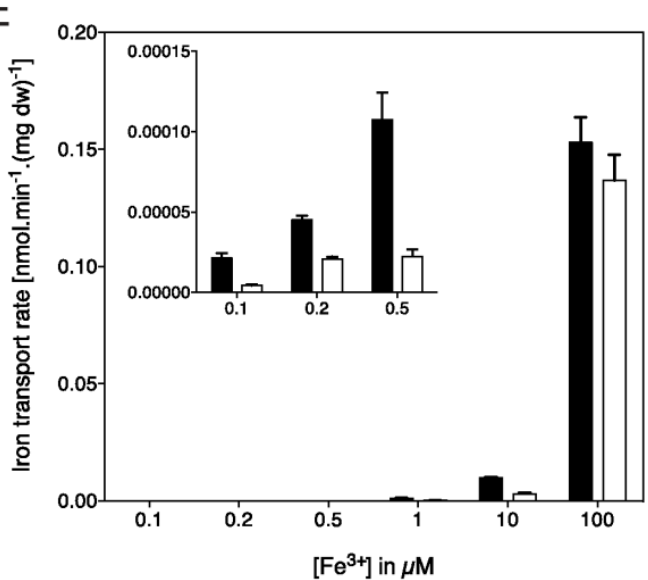

B

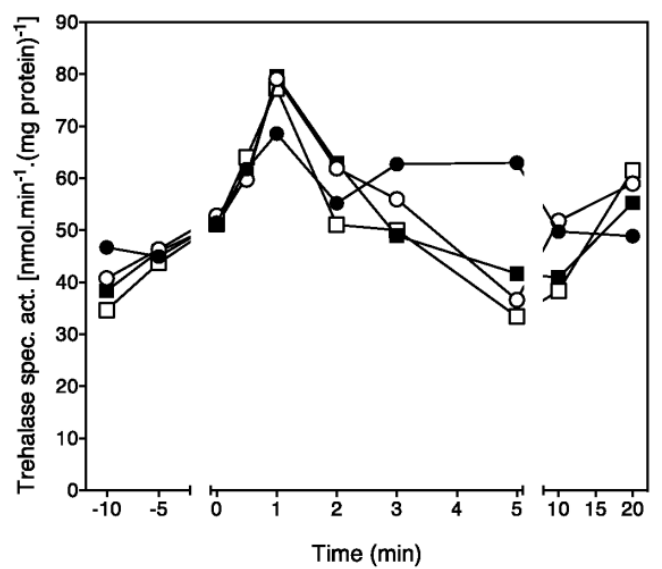

D

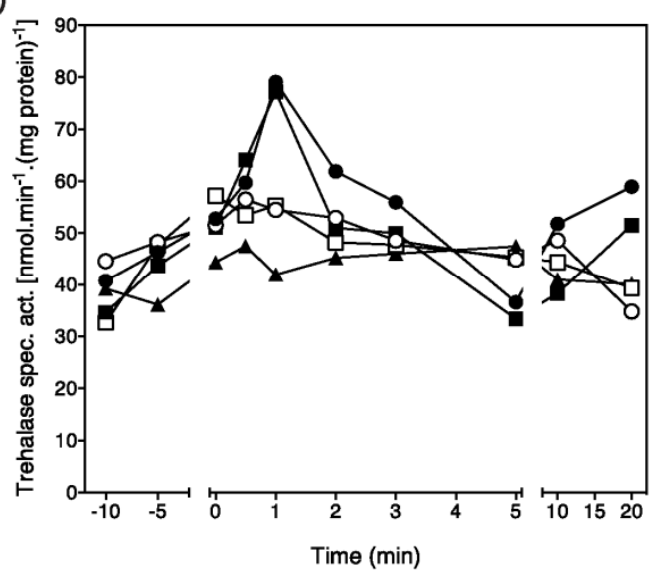

F

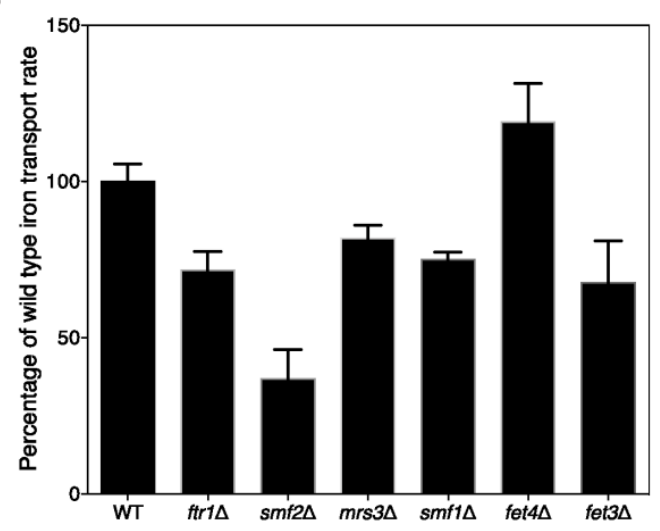

FIGURE 4: Re-addition of iron to iron-deprived cells triggers Ftr1-dependent activation of the PKA target trehalase. (A) Activation of trehalase in the wild type strain after addition of different concentrations of $\mathrm{FeCl}_{3}$ to cells deprived for iron for two days in the presence of the BPS chelator. $100 \mathrm{nM}$ (closed circles), $1 \mu \mathrm{M}$ (closed squares), $10 \mu \mathrm{M}$ (open circles) and $100 \mu \mathrm{M}$ (open squares) FeCl 3 . (B) Activation of trehalase in the wild type strain after addition of different concentrations of $\mathrm{FeCl}_{2}$ to cells deprived for iron for two days in the presence of the BPS chelator. $100 \mathrm{nM}$ (closed circles), $1 \mu \mathrm{M}$ (closed squares), $10 \mu \mathrm{M}$ (open circles) and $100 \mu \mathrm{M}$ (open squares) FeCl. (C) Activation of trehalase in wild type, ftr1 $\Delta$ and fet $3 \Delta$ strains after addition of different concentrations of $\mathrm{FeCl}_{3}$ to cells deprived for iron for two days in the presence of the BPS chelator. $100 \mu \mathrm{M}$ and $1 \mu \mathrm{M}$. Wild type, $1 \mu \mathrm{M}$ (closed circles) and $100 \mu \mathrm{M}$ (closed squares) FeCl $\mathrm{F}_{3}$ fr $1 \Delta, 1 \mu \mathrm{M}$ (open circles) and $100 \mu \mathrm{M}$ (open squares) $\mathrm{FeCl}_{3}$ and fet $3 \Delta, 100 \mu \mathrm{M} \mathrm{FeCl}$ (closed triangles). (D) Activation of trehalase in wild type, ftr1 $\Delta$ and fet $3 \Delta$ strains after addition of different concentrations of $\mathrm{FeCl}_{2}$ to cells deprived for iron for two days in the presence of the BPS chelator. Wild type, $1 \mu \mathrm{M}$ (closed circles) and $100 \mu \mathrm{M}$ (closed squares) $\mathrm{FeCl}_{2} ;$ ftr1 $\Delta, 1 \mu \mathrm{M}$ (open circles) and $100 \mu \mathrm{M}$ (open squares) $\mathrm{FeCl}_{2}$ and fet3 $\Delta, 100 \mu \mathrm{M}$ FeCl ${ }_{2}$ (closed triangles). (A-D) All experiments were performed 3-5 times with consistent results; representative results are shown. (E) Uptake of different concentrations of $\mathrm{Fe}^{55} \mathrm{Cl}_{3}$ in the wild type (black bars) and $f t r 1 \Delta$ (white bars) strains, $\mathrm{n}=2$. The inset shows the uptake at the lower concentrations in enlarged format. (F) Uptake of $100 \mu \mathrm{M} \mathrm{Fe}{ }^{55} \mathrm{Cl}_{3}$ in strains with a deletion of a single metal ion transporter gene, expressed as \% of the uptake in the wild type strain, $\mathrm{n}=2$. 
Zinc-induced signaling does not depend on intracellular zinc entry

Next, we investigated whether zinc-induced activation of trehalase originates from the Zrt1 transporter in the plasma membrane, similar to the involvement of Ftr1 in ironinduced signaling. Hence, we compared uptake of $\mathrm{Zn}^{65} \mathrm{Cl}_{2}$ at different concentrations of zinc in the wild type and $z r t 1 \Delta$ strain. At all concentrations uptake of zinc in the zrt1 $1 \Delta$ strain was strongly reduced and it was virtually absent at the lower concentrations (Fig. 5D). This indicates that Zrt1 is the dominant zinc transporter under our zinc deprivation conditions. However, while at low zinc concentrations, uptake was almost absent in the $\operatorname{zrt1} \Delta$ strain, there was still a significant level of zinc uptake present at the higher zinc concentrations of $100 \mu \mathrm{M}, 500 \mu \mathrm{M}$ and 1 $\mathrm{mM} \mathrm{ZnCl} 2$. Moreover, for $500 \mu \mathrm{M}$ and $1 \mathrm{mM} \mathrm{ZnCl}_{2}$ this uptake activity was actually higher than the zinc uptake activity of the wild type strain with $1 \mu \mathrm{M} \mathrm{ZnCl}_{2}$ (Fig. 5D). While addition of $1 \mu \mathrm{M} \mathrm{ZnCl}_{2}$ in the wild type strain triggered a strong increase in trehalase activity, addition of $100 \mu \mathrm{M}$ or
A

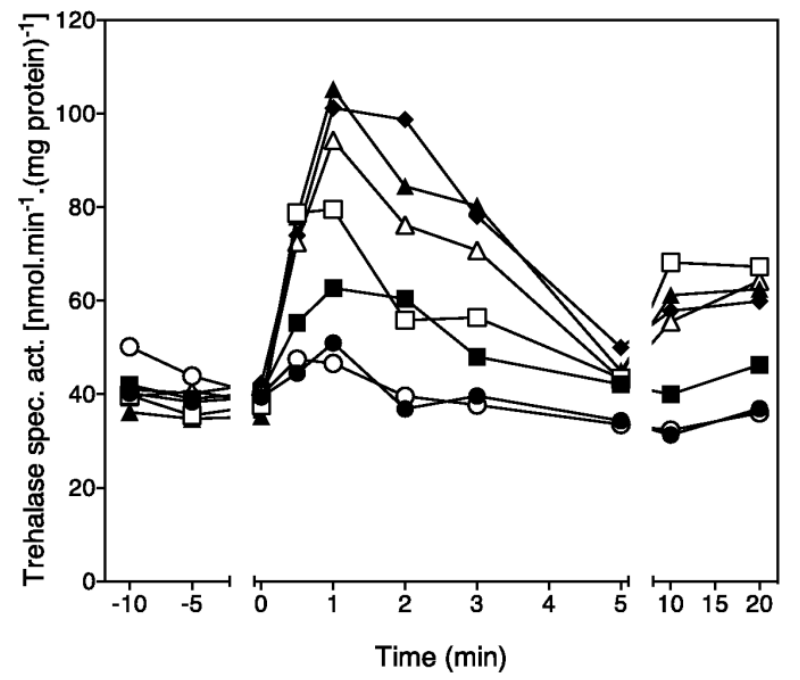

C

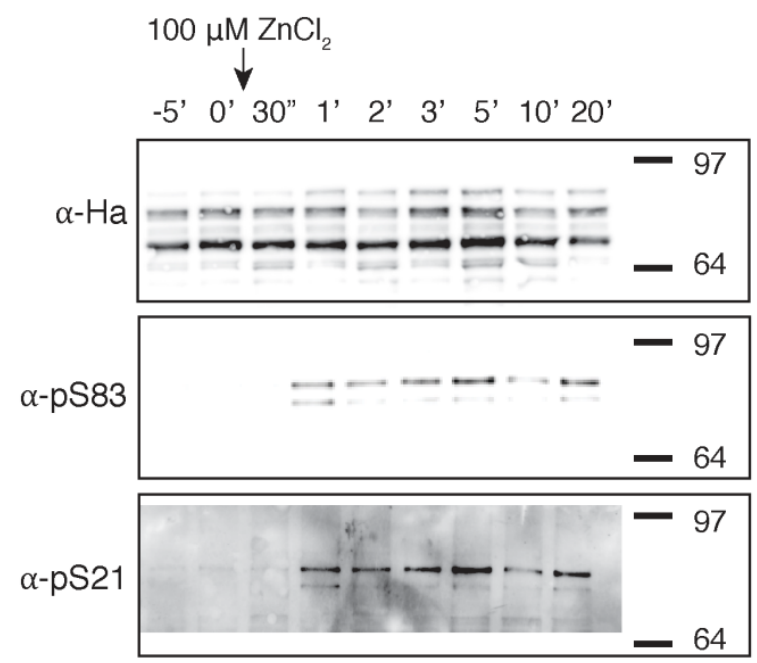

$\mathrm{B}$

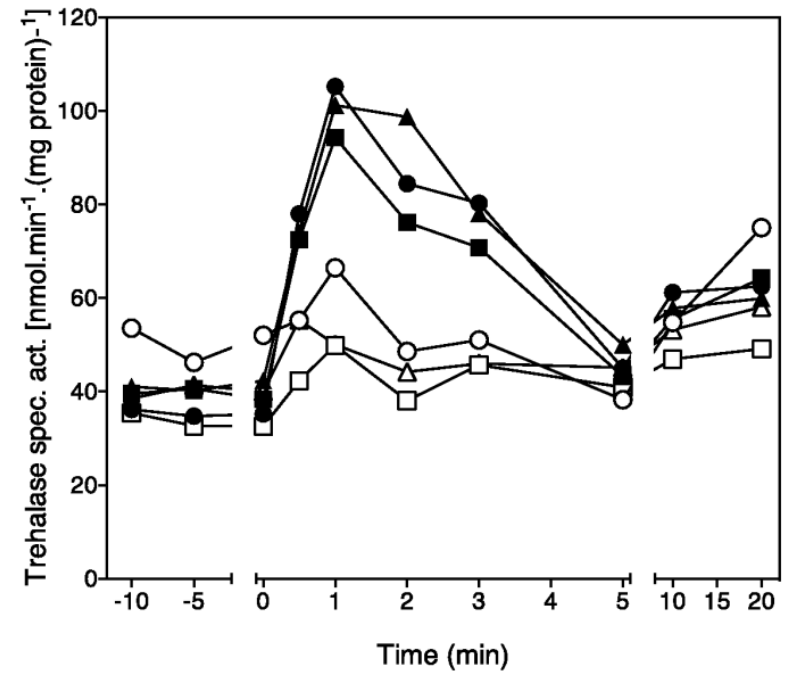

D

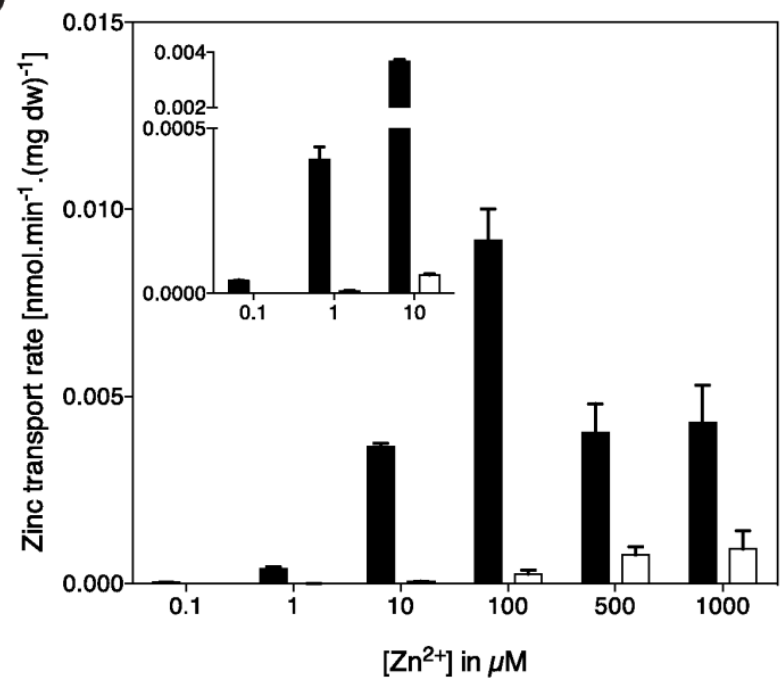

FIGURE 5: Re-addition of zinc to zinc-deprived cells triggers Zrt1-dependent activation of the PKA target trehalase. (A) Activation of trehalase in the wild-type strain after addition of different concentrations of $\mathrm{ZnCl}_{2}$ to cells deprived for zinc for two days in the presence of the EDTA chelator. $100 \mathrm{nM}$ (open circles), $500 \mathrm{nM}$ (closed squares), $1 \mu \mathrm{M}$ (open squares), $10 \mu \mathrm{M}$ (closed triangles), $100 \mu \mathrm{M}$ (open triangles), 1 $\mathrm{mM}$ (closed diamonds) $\mathrm{ZnCl}_{2}$ and starvation medium as negative control (closed circles). (B) Activation of trehalase in wild-type (closed symbols) and zrt1 $\Delta$ (open symbols) strains after addition of different concentrations of $\mathrm{ZnCl}_{2}$ to cells deprived for zinc for two days in the presence of the EDTA chelator. $1 \mu \mathrm{M}$ (circles), $100 \mu \mathrm{M}$ (squares) and $1 \mathrm{mM}$ (triangles). (A,B) All experiments were performed 3-5 times, representative results are shown. (C) Phosphorylation of trehalase on the amino acid residues S21 and S83 as detected with phosphospecific antibodies in response to addition of $100 \mu \mathrm{M} \mathrm{ZnCl}_{2}$ to cells of the wild type strain deprived for zinc for two days in the presence of the EDTA chelator. The trehalase-HA construct was expressed from the pYX212-NTH1-HA (LEU2) plasmid in the BY4742-strain. (D) Uptake of different concentrations of $\mathrm{Zn}^{65} \mathrm{Cl}_{2}$ in the wild type (black bars) and $z r t 1 \Delta$ strains (white bars), $\mathrm{n}=2$. The inset shows the uptake at the lower concentrations in enlarged format. 
$1 \mathrm{mM}$ in the zrt1 $\Delta$ strain only triggered very small residual increases in trehalase activity (Fig. 5B). Hence, as in the case of iron-induced signaling, we can conclude that entry of zinc into the cytosol is not enough to trigger signaling to the PKA pathway and that therefore the signal likely originates from the Zrt1 carrier in the plasma membrane.

Mutagenesis of specific aspartate residues affects Zrt1 function, but was unable to fully uncouple transport and signaling capacity

In an attempt to obtain further evidence that Zrt1 acts as a transceptor for zinc-induced signaling to the PKA pathway, we have performed site-directed mutagenesis with the aim of specifically affecting either transport or signaling. We have focussed specifically on negatively charged amino acid residues in or near transmembrane domains since they may be involved in the binding and translocation of the positive zinc ions through the membrane. We have changed the aspartate (D) and glutamate (E) residues indicated in Fig. 6A into uncharged asparagine $(\mathrm{N})$ and glutamine $(\mathrm{Q})$ residues, respectively. Signaling capacity and zinc uptake activity were measured in a $\operatorname{zrt1} \Delta$ strain expressing the mutant alleles from a plasmid or harboring the empty vector. In most cases the strains expressing a mutant Zrt1 allele displayed a similar extent of zinc-induced activation of trehalase compared to the strain expressing the wild

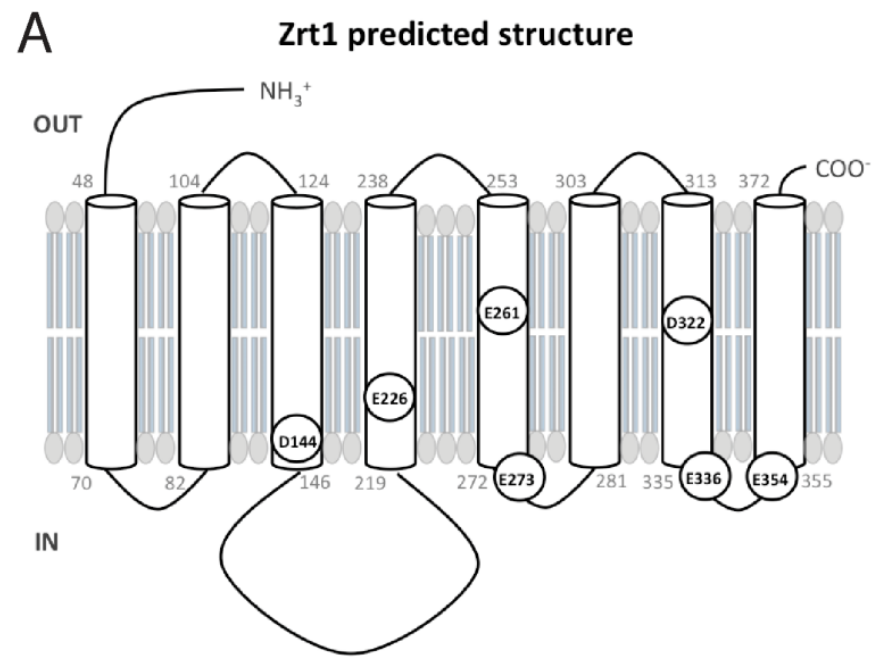

$\mathrm{B}$

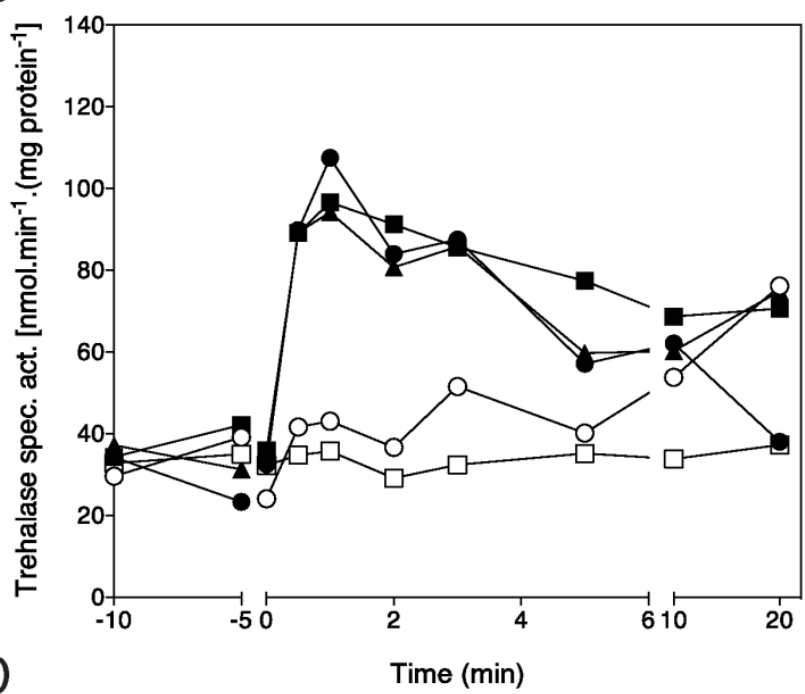

C

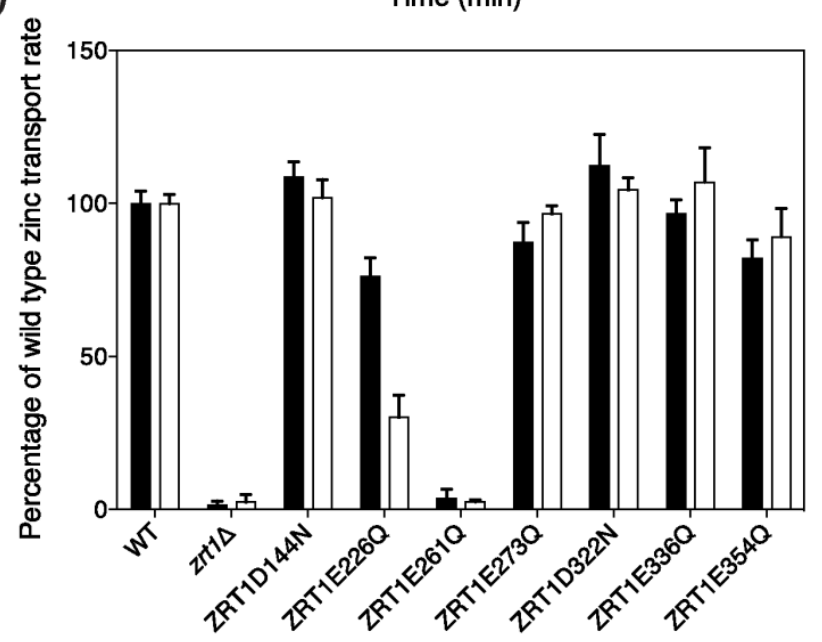

Time (min)

FIGURE 6: Signaling capacity and zinc uptake activity in a zrt1 $\Delta$ strain expressing different Zrt1 alleles containing a mutated aspartate or glutamate residue. (A) Schematic overview of Zrt1 with the location in or close to TMDs indicated of the aspartate and glutamate residues that were mutagenized to asparagine or glutamine, respectively. (B, C) Activity of trehalase as a function of time after addition of $100 \mu \mathrm{M}$ $\mathrm{ZnCl}_{2}$ to cells of a zrt1 $\Delta$ strain harboring the wild type ZRT1 gene on plasmid YCplac33-Zrt1, the empty plasmid YCplac33 or different point mutant alleles of ZRT1 on plasmid YCplac33. All experiments were performed 3-5 times; representative results are shown. (B) Wild type ZRT1 (closed circles), zrt1 (open circles), ZRT1 ${ }^{\mathrm{D} 144 \mathrm{~N}}$ (closed squares), $Z R T 1^{\mathrm{E2260}}$ (closed triangles) and $Z R T 1^{\mathrm{E2} 2610}$ (open squares). (C) Wild type $Z R T 1$

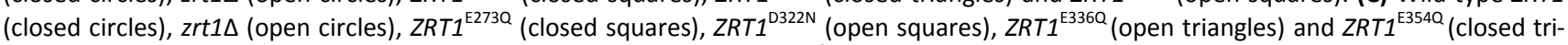
angles). (D) Uptake of either $10 \mu \mathrm{M}$ (black bars) or $100 \mu \mathrm{M}$ (white bars) $\mathrm{Zn}^{65} \mathrm{Cl}_{2}$ in the same strains as used in $\mathbf{B}, \mathbf{C}, \mathrm{n}=2$. 
type allele, except for the E261Q allele, which was completely deficient in supporting trehalase activation (Fig. 6B, C). The latter strain was also completely deficient in $\mathrm{Zn}^{65}$ uptake, similar to the zrt1 $\Delta$ strain (Fig. 6D). This identifies the E261 glutamate residue, located in the middle of TMD5, as a crucial residue for Zrt1 functionality, both for transport and signaling. Interestingly, the strain expressing the E226Q allele displayed a partial reduction in zinc uptake activity with about $70 \%$ at a concentration of $100 \mu \mathrm{M}$ $\mathrm{ZnCl}_{2}$ (Fig. 6D), while it showed the same extent of trehalase activation as the strain expressing the wild type allele (Fig. 6B). Hence, with the E226Q allele we observed a partial uncoupling of transport and signaling activity of Zrt1.

\section{DISCUSSION}

We have introduced the concept of transceptors for transporters with an additional receptor function that is linked to the transport function, in the sense that transport of the substrate through the transceptor elicits a conformational change that triggers a signaling event to the inside of the cell [23]. This contrasts with signaling events that are initiated after the entry of the transporter substrate into the cell and in which the transporter only plays a role by making the substrate available for sensing by an intracellular sensor system. In recent years, more and more examples of transceptors or putative transceptors in other organisms have been reported. In all cases it concerned nutrient transceptors. The CDT-1,CDT-2 and CLP1 genes have recently been shown to encode three putative cellodextrin transceptors in the mold Neurospora crassa [40, 41] and the Hxt1 monosaccharide transporter been shown to have an additional sensor function in the smut Ustilago maydis [42]. Plant transceptors include a set of nitrate transceptors encoded by LATD/NIP [43, 44], MtNRT1,3 [45, 46], AtNRT1.1/CHL1 [47-50] and AtNRT1.2 [47, 51], as well as putative ammonium transceptors such as LjAMT3 [52], AtAMT1,3 [53] and AtAMT1.1 [54], and a putative sulfate transceptor AtSULTR1;2 [55, 56]. Transceptors have been identified in other organisms, such as GT1 in Leishmania mexicana [57] and GLUT2 in mice [58-60]. Finally, the concept of transceptors has also emerged in human cells with the identification of hCNT1 [61] and hSNAT2 [62-64]. Hence, the concept of transceptors seems to be conserved throughout evolution in eukaryotic organisms.

A major difference between the transceptor system that we discovered in yeast for nutrient-induced activation of the PKA signaling pathway and the other transceptor systems reported in the literature, is that in our system multiple transceptors for different nutrients signal to the same target pathway, the PKA pathway. This is a classical signal transduction pathway that is not directly related to the transport or the metabolism of the nutrient concerned, but rather controls a variety of physiological and developmental properties of yeast cells. Our current discovery of Ftr1 and Zrt1 acting as transceptors for the micronutrients iron and zinc, respectively, makes it very likely that all these transceptors use the same signaling mechanism for activation of the PKA pathway. This should facilitate its elucidation. Another common characteristic of the transceptors acting on the PKA pathway is that the high sensitivity for signaling by the nutrient matches with the high affinity for transport of the nutrient, suggesting that the goal of the signaling is related to the function of the ligand as a nutrient. Apparently, the transceptor signals to the cellular machinery that a deficient essential nutrient has become available again and that the fermentable growth medium is thus again complete. Since this provides the most optimal growth medium for the species $S$. cerevisiae, fermentation and cell proliferation should be stimulated and storage sugar and stress tolerance mechanisms downregulated. The cellular machinery and physiology should be focussed on generating offspring as rapidly as possible.

Iron- and zinc-induced activation of trehalase was weaker and also more transient compared to activation with the macronutrients nitrogen, phosphate and sulfate. This may have to do with the extent to which the cells can enter stationary phase and therefore acquire a low-PKA status upon iron or zinc deprivation. Since all the other nutrients are still present, and since all major nutrients are well-established ligands for activation of the PKA pathway, starvation for only iron or only zinc in some way has to be able to shut off activation of PKA by all the other nutrients. Starvation for a macronutrient may be more powerful in this respect. We also know from extensive previous work on optimizing the starvation conditions required for observation of subsequent maximal nutrient-induced activation of trehalase that deep entrance into G0 with a clear low PKA phenotype is essential for a pronounced subsequent response. This may explain why it was more difficult to establish the proper starvation conditions for iron and zinc than with the macronutrients nitrogen, phosphate and sulfate. Another possible explanation is the high toxicity of metal ions and thus the need to curtail metal ion influx into the cytosol to avoid accumulation of excessive levels of metal ions. A similar rapid and transient activation of trehalase was observed after addition of ammonium to nitrogen-starved cells [5], which may be related in a similar way to the possible detrimental effect of ammonium on the intracellular $\mathrm{pH}$.

The high toxicity of metal ions was a possible concern for the interpretation of our results. However, the data in Fig. $3 \mathrm{~A}$ show that addition of $100 \mu \mathrm{M}$ of either $\mathrm{FeCl}_{3}$ or $\mathrm{ZnCl}_{2}$ to appropriately starved cells caused resumption of growth, contradicting a strong toxic effect under the conditions in which we measured signaling and transport. Also, short-term, relatively simple responses like posttranslational trehalase activation may be less sensitive to high levels of metal ions than more complex, long-term responses like gene expression or physiological read-outs.

Because the response to re-addition of the micronutrients iron and zinc was less pronounced than with the macronutrients nitrogen, phosphate and sulfate, we have concentrated on trehalase activation as a read-out for activation of the PKA pathway, rather than longer-term responses, like changes in gene expression and in physiological properties, that have previously been used for that pur- 
pose. Nutrient-induced trehalase activation is both the most sensitive, the most rapid and most specific target of PKA activity known in yeast. The mechanism involved has been elucidated in great detail and it is very well established that the increase in trehalase activity is dependent on phosphorylation by PKA [15, 65-67].

The main goal of the present paper was to elucidate whether the high-affinity transporters Ftr1 for iron and Zrt1 for zinc, acted as transceptors for iron- and zincinduced activation of the PKA pathway in appropriately starved cells, as previously demonstrated for the highaffinity transporters Gap1 [17], Mep2 [5], Pho84 [19] and Sul1,2 [22] for amino acid-, ammonium-, phosphate- and sulfate-induced activation in appropriately starved cells. To achieve this goal, different experimental approaches were tried in order to uncouple signaling from transport, in the sense that signaling still happens without entry of the substrate into the cytosol or that the substrate enters into the cytosol through another way, without signaling being triggered. The finding that deletion of Ftr1 or Zrt1 eliminated iron- or zinc-induced signaling, respectively, argues against involvement of a specific receptor for the metal ions, as was previously found for glucose activation of the CAMPPKA pathway by the glucose- and sucrose-sensing Gprotein coupled receptor Gpr1 [68]. The next challenge was to discriminate between the requirement of Ftr1 and Zrt1 as transporter-receptors, initiating the signaling upon transport of the metal ion through the protein transmembrane passageway, or merely as regular transporters that have to bring the metal ion into the cell so that it can be sensed by an intracellular sensing mechanism.

The finding that for high iron concentrations, the uptake in the ftr1 $\Delta$ strain was not significantly different from that in the wild type strain, indicated that Ftr1 itself rather than intracellular iron acted as trigger for the signaling. This is consistent with all previous work on the nutrient transceptors involved in activation of the PKA pathway in yeast that the transceptor itself acts as a nutrient receptor and that the nutrient does not have to enter the cytosol to trigger signaling [1]. Our results confirm earlier observations that in addition to Ftr1 other non-specific iron uptake mechanisms are also upregulated under iron deprivation conditions [31]. This explains why Ftr1 is not essential for uptake of high levels of iron under our conditions. The Fet3 dependency of signaling is likely due to the requirement of Fet3 for proper localization of Ftr1 at the plasma membrane. In principle, we cannot exclude the possibility that Fet3 itself would act as the iron sensor. However, this seems rather unlikely since it functions as an oxidoreductase and the other transceptors for activation of the PKA pathway in yeast, including Zrt1, do not require such an enzyme for functionality. A role of Ftr1 as iron sensor, on the other hand, fits very well with the thoroughly established transceptor concept for activation of the PKA pathway by the other nutrients.

We found that re-addition of both $\mathrm{Fe}^{2+}$ and $\mathrm{Fe}^{3+}$ to ironstarved cells could trigger trehalase activation with equal efficiency. This was surprising as it was suggested in the current model for high-affinity uptake that $\mathrm{Fe}^{2+}$ is oxidized by $\mathrm{Fet} 3$ to $\mathrm{Fe}^{3+}$ and subsequently channelled inside the protein complex to Ftr1 for uptake [30]. Hence, in this model, $\mathrm{Fe}^{2+}$ itself does not act as a substrate for Ftr1. Our finding might imply that $\mathrm{Fe}^{3+}$ could directly act as a signaling substrate of Ftr1, although we cannot exclude that it is first reduced to $\mathrm{Fe}^{2+}$ by iron reductases like Fre1 present at the plasma membrane, before being oxidized back to $\mathrm{Fe}^{3+}$ by Fet 3 and channelled to Ftr 1 for uptake and signaling. Hence, for uptake $\mathrm{Fe}^{2+}$ may have to be oxidized to $\mathrm{Fe}^{3+}$, while for signaling it might not be required. Although previous work showed that for the Gap1 and Pho84 transceptors, the same substrate binding site is used for transport and signaling, other data using non-transported substrate analogues showed that complete transport is not required for signaling $[18,20,69]$.

To establish Zrt1 as a zinc transceptor, we could use a similar argumentation as in the case of Ftr1. Although in this case, deletion of Zrt1 reduced zinc uptake at all concentrations, there was a level of uptake left in the zrt1 strain at the higher concentrations that was similar to the level of zinc uptake at lower concentrations in the wild type strain. Since the latter were able to trigger trehalase activation as opposed to the high concentrations of zinc in the $z r t 1 \Delta$ strain, we could conclude that the signaling is not triggered by intracellular zinc but rather by Zrt1 acting as a transceptor at the level of the plasma membrane. As opposed to iron signaling by Ftr1, deletion of ZRT1 did not completely abolish zinc-induced trehalase activation. This might indicate that another zinc transporter might also have limited transceptor functionality. A possible candidate is the low-affinity zinc transporter Zrt2, which shows strong structural and sequence similarity with Zrt1 [9]. In the presence of Zrt1, Zrt2 does not influence high-affinity uptake and is not expressed under zinc-limiting conditions. However, upon deletion of ZRT1, expression of ZRT2 might be enhanced under zinc-limiting conditions in order to cope with the zinc limitation and then act as a weak transceptor upon re-addition of zinc to the starved cells.

Finally, we have obtained more insight into the action mechanism of Zrt1 through mutational analysis. We found that the $\mathrm{Zrt}^{\mathrm{E} 261 \mathrm{Q}}$ protein completely lacked signaling and transport capacity. $\mathrm{Glu}^{261}$ is a strongly conserved residue in metal ion transporters. Previous work has shown that mutagenesis of the corresponding residue, $\mathrm{Glu}^{228}$, in the Arabidopsis thaliana IRT1 metal ion transporter, another member of the ZIP family, completely abolished transport for all metal ions that are transported by this carrier [70]. Hence, this residue may be essential for proper functioning of all ZIP family members. Our mutational analysis also identified a mutant allele, $\mathrm{Zrt1}^{\mathrm{E} 226 \mathrm{Q}}$, in which zinc uptake was partially reduced with about $70 \%$ but signaling with the same concentration of $\mathrm{ZnCl}_{2}$ was not affected. This partial uncoupling of transport and signaling provides further support for Zrt1 functioning as a transceptor, since it argues against the substrate in the cytosol being responsible for triggering the signaling. It is in agreement with previous results obtained with the other yeast transceptors in which transport could be abolished without affecting signaling $[18,21,22]$. Mutagenesis of specific putative proton 
binding sites in Sul1, Sul2 and Pho84 abolished transport but not signaling indicating that binding of the substrate to the transporter is enough to establish the conformation that triggers signaling. $\mathrm{Glu}^{226}$ is also strongly conserved in ZIP family members [70], but its importance has not been addressed yet by site-directed mutagenesis. $\mathrm{Glu}^{226}$ is located in TMD IV, closer to the border with the cytosol than $\mathrm{Glu}^{261}$ in TMD V. This may allow the mutant transceptor $\mathrm{Zrt1}^{\mathrm{E226Q}}$ to bind zinc and gain the conformation that triggers signaling to the PKA pathway without being able to transport the zinc ion efficiently further into the cytosol.

In conclusion, our work has identified yeast Ftr1 and Zrt1 as the first transceptors for micronutrients, iron and zinc, respectively. We have shown that deprivation for iron or zinc leads to growth arrest with development of a lowPKA phenotype and that re-addition of iron or zinc to cells starved for iron or zinc, respectively, triggers rapid activation of the well-established PKA target trehalase. The identification of Ftr1 and Zrt1 as micronutrient transceptors strengthens the concept that yeast cells use the highaffinity transporters that are generally induced upon starvation for essential nutrients as receptors upon exit from the starved stationary phase. Our finding makes it likely that all transceptors use the same or a similar signaling mechanism for activation of the PKA pathway and its elucidation may be facilitated by the current availability of a wide range of transceptors.

\section{MATERIALS AND METHODS}

\section{Yeast strains}

The S. cerevisiae strains used in this work are shown in Table 1. With exception of those used for the results in Fig. 4F, they are all isogenic with wild type strain 51278 (MATa ura3A). The deletion strains have been created by transferring the KanMX cassette from the strains in the Euroscarf deletion collection to the $\Sigma 1278$ wild type strain. Primers used for amplification of the cassette were Ftr1 Fw (ACTACTAACTCCTGAGACA) Ftr1 Rv (ATGGTTATATCCTGGCATG), Fet3 Fw (GCTTGCCTATTTCACGGTTAC), Fet3 Rv (TCTCAGTAATCTCAGGCTATT), Zrt1 Fw (AAATGCACTAGAACATGGCG) and Zrt1 Rv (TTCATGACTATTTAAATGCCTT). The strains used for the results in Fig. 4F

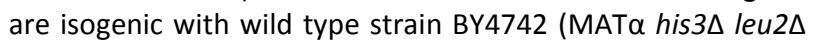
lys $2 \Delta$ ura $3 \Delta$ ) and with the transporter deletion strains from the Euroscarf deletion collection.

\section{Growth media and starvation conditions}

To obtain iron- or zinc--starved cells, the cells were first cultured at $30^{\circ} \mathrm{C}$ into exponential phase $\left(\mathrm{OD}_{600 \mathrm{~nm}}=1.5-2\right)$ in all trace medium with $2 \%(\mathrm{w} / \mathrm{v})$ glucose. The composition of all trace medium is based on CSM complete medium and contains the list of chemicals shown in Table 2. The medium was prepared by mixing all components except vitamins together in milliQ water and then autoclaved. A 500x filter-sterilized vitamin stock was prepared separately and the appropriate amount added to the medium right before use. Exponentialphase cells were harvested and suspended in starvation medium supplemented with a specific chelator and containing $4 \%$ glucose. They were incubated under shaking for 24,48 or $72 \mathrm{~h}$ at $30^{\circ} \mathrm{C}$. Care was taken that the glucose level remained high $(>2 \%)$ throughout the incubation. For iron starvation medium, ferric chloride was omitted from the all trace medium and the medium was supplemented with $80 \mu \mathrm{M}$ BPS or $500 \mu \mathrm{M}$ ferrozine. For zinc starvation medium, zinc sulfate was excluded from the all trace medium and the medium was supplemented with $1 \mathrm{mM}$ EDTA and $10 \mathrm{mM}$ citrate.

\section{Growth curves and budding percentage}

$100 \mathrm{ml}$ cultures were grown under continuous shaking at $30^{\circ} \mathrm{C}$, in the indicated medium. At regular time intervals, a $1 \mathrm{ml}$

Table 1. S. cerevisiae strains used in this study.

\begin{tabular}{|c|c|c|}
\hline Strain name & Genotype & Origin/Reference \\
\hline$\Sigma 1278(\mathrm{JT4500})$ & 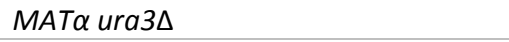 & ResGen/Invitrogen \\
\hline JSO01 & $\Sigma 1278$ MAT $\alpha$ ura $3 \Delta$ ftr1::KanMX & This study \\
\hline JSO02 & $\Sigma 1278$ MAT $\alpha$ ura3A zrt1::KanMX & This study \\
\hline JSO03 & $\Sigma 1278$ MAT $\alpha$ ura3A ctr1::KanMX & This study \\
\hline JSO04 & 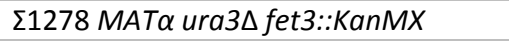 & This study \\
\hline JS005 & 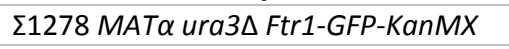 & This study \\
\hline JS006 & 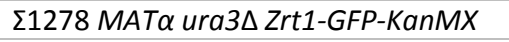 & This study \\
\hline JS007 & $\Sigma 1278$ MAT $\alpha$ ura3A Ctr1-GFP-KanMX & This study \\
\hline JS008 & 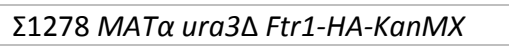 & This study \\
\hline JSO09 & 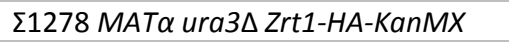 & This study \\
\hline JS010 & 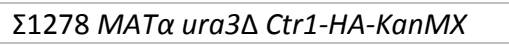 & This study \\
\hline JS011 & 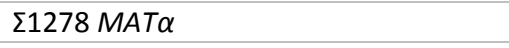 & This study \\
\hline \multirow[t]{7}{*}{ BY4742 } & $M A T \alpha$ his $3 \Delta$ leu2 2 lys $2 \Delta$ ura3 $\Delta$ & Euroscarf \\
\hline & $B Y 4742 \mathrm{ftr} 1 \Delta$ & Euroscarf \\
\hline & $B Y 4742$ smf $2 \Delta$ & Euroscarf \\
\hline & $B Y 4742 m r s 3 \Delta$ & Euroscarf \\
\hline & BY4742 smf1D & Euroscarf \\
\hline & BY4742 fet4 $\Delta$ & Euroscarf \\
\hline & 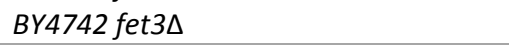 & Euroscarf \\
\hline
\end{tabular}


sample was taken for $\mathrm{OD}_{600 \mathrm{~nm}}$ measurement with a Biophotometer from Eppendorf.

\section{RNA extraction and qPCR}

For determination of FTR1 and ZRT1 expression, appropriately-starved cells were collected, spun down, and the pellets were frozen in liquid nitrogen and stored at $-80^{\circ} \mathrm{C}$. Total RNA was isolated by phenol extraction and treated with RNAse free DNAse (Roche). cDNA was prepared following the instructions of the Reverse Transcription Kit by Promega A3500. Subsequently, relative quantification of FTR1/ZRT1 expression and the reference genes 185 and $A C T 1$ was performed using realtime PCR with a GoTaq qPCR Master Mix from Promega for a StepOnePlus System (Applied Biosystems). The primers used were: Fw Ftr11 (CCTTGTCTGTGGCGACGTT), Rv Ftr1 (CTACGAACTTCCCGAGCAAACTA), Fw Zrt1 (GCCATCGGTTTGGGTGTTC), Rv Zrt1 (CCAGAGATAACAAGCGCAGTGT).

\section{Fluorescence microscopy}

The fluorescence-based localization studies of Ftr1-GFP and Zrt1-GFP were performed with iron or zinc-starved cells, respectively, and carried out using an Olympus FV1000 confocal laser scanning microscope. Images were processed with the accompanying software, FV10-ASW 2.0.

\section{Biochemical determinations}

Trehalose and glycogen content were determined using previously described protocols, in which trehalose and glycogen are converted to glucose by trehalase and $\beta$-amyloglucosidase, respectively and subsequently determined using the GOD-PAP method [17]. Trehalase activity after addition of iron or zinc was determined as previously described [17]. The specific activity of trehalase is expressed as nmol glucose liberated* $\min ^{-1} *(\mathrm{mg} \text { protein })^{-1}$. Total amount of protein in the samples was determined using the standard Lowry method.

\section{Determination of iron and zinc uptake}

Cells were starved either for iron using the BPS chelator, or for zinc using the EDTA chelator as described above. Subsequently, cells were washed once with $25 \mathrm{mM}$ MES buffer pH 6.0 and resuspended in starvation medium (without chelators) to a density of $60 \mathrm{mg} / \mathrm{mL}$. After acquiring blank samples, $40 \mu \mathrm{L}$ of cells were incubated for each sample in 3-fold for $10 \mathrm{~min}$ at $30^{\circ} \mathrm{C}$. Subsequently, $10 \mu \mathrm{L}$ of a $5 \mathrm{x}$ concentrated stock of the indicated concentration of $\mathrm{FeCl}_{3}$ (containing a specific activity of $3,000 \mathrm{cpm} / \mathrm{nmol} \mathrm{Fe}^{55}$ ) or $\mathrm{ZnCl}_{2}$ (containing a specific activity of $3,000 \mathrm{cpm} / \mathrm{nmol} \mathrm{Zn}^{65}$ ) was added to the samples. The samples were incubated for $1 \mathrm{~min}$ in the presence of the nutrient after which uptake was stopped by addition of $10 \mathrm{~mL}$ ice-cold unlabelled $\mathrm{FeCl}_{3}$ or $\mathrm{ZnCl}_{2}$ equal to the highest concentration used in the experiment. The sample was filtered over a glass microfiber filter (Whatman GF/C) prewet with the unlabelled $\mathrm{FeCl}_{3}$ or $\mathrm{ZnCl}_{2}$. The filter was immersed in scintillation fluid in a scintillation tube and the $\mathrm{Fe}^{55}$ or $\mathrm{Zn}^{65}$ content was determined using a Hidex 300 SL Liquid Scintillation Counter. Uptake was expressed as $\mathrm{nmol}^{*} \mathrm{~min}^{-1} *(\mathrm{mg} \text { dry weight })^{-1}$.

\section{Site-directed mutagenesis}

To obtain the point mutant forms of Zrt1, site-directed mutagenesis was used. Full-length ZRT1 was cloned into the

Table 2. Composition of all trace medium.

\begin{tabular}{|c|c|c|}
\hline Component & Concentration & Component \\
\hline Ammonium sulfate & $5 \mathrm{~g} / \mathrm{L}$ & Ammonium sulfate \\
\hline Biotin & $0.002 \mathrm{mg} / \mathrm{L}$ & Biotin \\
\hline Calcium pantothenate & $0.4 \mathrm{mg} / \mathrm{L}$ & Calcium pantothenate \\
\hline Folic acid & $0.002 \mathrm{mg} / \mathrm{L}$ & Folic acid \\
\hline Inositol & $2 \mathrm{mg} / \mathrm{L}$ & Inositol \\
\hline Niacin & $0.4 \mathrm{mg} / \mathrm{L}$ & Niacin \\
\hline P-aminobenzoic acid & $0.2 \mathrm{mg} / \mathrm{L}$ & P-aminobenzoic acid \\
\hline Pyridoxine hydrochloride & $0.4 \mathrm{mg} / \mathrm{L}$ & Pyridoxine hydrochloride \\
\hline Riboflavin & $0.2 \mathrm{mg} / \mathrm{L}$ & Riboflavin \\
\hline Thiamine hydrochloride & $0.4 \mathrm{mg} / \mathrm{L}$ & Thiamine hydrochloride \\
\hline Boric acid & $0.5 \mathrm{mg} / \mathrm{L}$ & Boric acid \\
\hline Copper sulfate & $0.04 \mathrm{mg} / \mathrm{L}$ & Copper sulfate \\
\hline Potassium iodide & $0.1 \mathrm{mg} / \mathrm{L}$ & Potassium iodide \\
\hline Ferric chloride (omitted in -Fe) & $0.2 \mathrm{mg} / \mathrm{L}$ & Ferric chloride (omitted in -Fe) \\
\hline Manganese sulfate & $0.4 \mathrm{mg} / \mathrm{L}$ & Manganese sulfate \\
\hline Sodium molybdate & $0.2 \mathrm{mg} / \mathrm{L}$ & Sodium molybdate \\
\hline Zinc sulfate (omitted in -Zn) & $0.4 \mathrm{mg} / \mathrm{L}$ & Zinc sulfate (omitted in -Zn) \\
\hline Magnesium sulfate & $1 \mathrm{~g} / \mathrm{L}$ & Magnesium sulfate \\
\hline Sodium chloride & $100 \mathrm{mg} / \mathrm{L}$ & Sodium chloride \\
\hline Potassium phosphate dihydrate & $1.75 \mathrm{~g} / \mathrm{L}$ & Potassium phosphate dihydrate \\
\hline Di-potassium hydrogen phosphate trihydrate & $0.25 \mathrm{~g} / \mathrm{L}$ & Di-potassium hydrogen phosphate trihydrate \\
\hline Calcium chloride & $120 \mathrm{mg} / \mathrm{L}$ & Calcium chloride \\
\hline Uracil & $50 \mathrm{mg} / \mathrm{L}$ & Uracil \\
\hline Glucose & $2 \%(w / v)$ & Glucose \\
\hline
\end{tabular}


Table 3. List of primers used for site-directed mutagenesis of Zrt1.

\begin{tabular}{|c|c|c|}
\hline Name & Mutation & Sequence \\
\hline Zrt1-D105N Fw & ZRT1 $^{\mathrm{D} 105 N}$ & CACTTAATGAACCCTGCTTAT \\
\hline Zrt1-D105N Rv & $\mathrm{ZRT1}^{\mathrm{D} 105 \mathrm{~N}}$ & ATAAGCAGGGTTCATTAAGTG \\
\hline Zrt1-D144N Fw & $\mathrm{ZRT1}^{\mathrm{D} 144 \mathrm{~N}}$ & TTCCTTACTAATCTATTCAGT \\
\hline Zrt1-D144N Rv & $\mathrm{ZRT1}^{\mathrm{D} 144 \mathrm{~N}}$ & ACTGAATAGATTAGTAAGGAA \\
\hline Zrt1-E226Q Fw & ZRT1 ${ }^{\text {E226Q }}$ & TTAATTTTACAATTCGGTGTG \\
\hline Zrt1-E226Q Rv & ZRT1 $^{\text {E226Q }}$ & CACACCGAATTGTAAAATTAA \\
\hline Zrt1-E261Q Fw & $\mathrm{ZRT}^{\mathrm{E} 261 \mathrm{Q}}$ & CAATCATTTCAAGGTTTAGGT \\
\hline Zrt1-E261Q Rv & $\mathrm{ZRT}^{\mathrm{E} 261 \mathrm{Q}}$ & ACCTAAACCTTGAAATGATTG \\
\hline Zrt1-E273Q Fw & ZRT1 $^{\text {E273Q }}$ & TCAGCCATTCAATTCCCTAGA \\
\hline Zrt1-E273Q Rv & ZRT1 $^{\text {E273Q }}$ & TCTAGGGAATTGAATGGCTGA \\
\hline Zrt1-D322N Fw & $\mathrm{ZRT1}^{\mathrm{D} 322 \mathrm{~N}}$ & GGTGTTTTGAATGCCATTTCT \\
\hline Zrt1-D322N Rv & $\mathrm{ZRT}^{\mathrm{D} 322 \mathrm{~N}}$ & AGAAATGGCATTCAAAACACC \\
\hline Zrt1-E336Q Fw & ZRT1 ${ }^{\text {E336Q }}$ & GGTTTGGTTCAACTACTAGCA \\
\hline Zrt1-E336Q Rv & ZRT1 $^{\text {E336Q }}$ & TGCTAGTAGTTGAACCAAACC \\
\hline Zrt1-E354Q Fw & $\mathrm{ZRT1}^{\mathrm{E} 354 \mathrm{Q}}$ & GATCTAAGACAATTGTCCTTC \\
\hline Zrt1-E354Q Rv & $\mathrm{ZRT1}^{\mathrm{E} 354 \mathrm{Q}}$ & GAAGGACAATTGTCTTAGATC \\
\hline
\end{tabular}

YCplac33 plasmid using the Sall (Fw) and Xmal (Rev) restriction sites using the primers Zrt1-Sal1 Fw TTTTTTTGTCGACAGACTTGAGATAGATGTACC and Zrt1-Xmal Rv TTTTTTCCCGGGAGTGGTCAATGAGATCAAA. Subsequently, site-directed mutagenesis was performed using primers containing the specific mutations as indicated in the primer list in Table 3 , in combination with the Q5 High Fidelity DNA Polymerase and a PCR reaction containing 18 cycles adjusted to the conditions used. This PCR amplification yielded a plasmid construct carrying ZRT1 with the desired mutation. The original plasmid was subsequently digested with $1 \mu \mathrm{L}$ of the Dpnl restriction enzyme, leaving only the newly synthesized plasmids in intact form. The newly constructed plasmids were subsequently transformed into competent $E$. coli cells, the transformants selected based on ampicillin resistance, the plasmids purified and finally transformed into the $\sum z r t 1 \Delta$ strain.

\section{Reproducibility of the results}

All experiments were repeated at least two times. Standard deviations are shown for comparisons between independent data points (transport measurements). Time-course experiments of metal-ion induced trehalase activation were repeated three to five times. Representative results are shown for

\section{REFERENCES}

1. Conrad M, Schothorst J, Kankipati HN, Van Zeebroeck G, RubioTexeira M, Thevelein JM (2014). Nutrient sensing and signaling in the yeast Saccharomyces cerevisiae. FEMS Microbiol Rev 38(2): 254-299.

2. Broach JR (2012). Nutritional control of growth and development in yeast. Genetics 192(1): 73-105.

3. Ye L, Berden JA, van Dam K, Kruckeberg AL (2001). Expression and activity of the Hxt7 high-affinity hexose transporter of Saccharomyces cerevisiae. Yeast 18(13): 1257-1267.

4. Jauniaux JC, Grenson M (1990). GAP1, the general amino acid permease gene of Saccharomyces cerevisiae. Nucleotide sequence, protein similarity with the other bakers yeast amino acid permeases, and nitrogen catabolite repression. Eur J Biochem 190(1): 39-44. these comparisons between collections of interdependent data points.

\section{CONFLICT OF INTEREST}

The authors declare no conflict of interest.

\section{COPYRIGHT}

(C) 2017 Schothorst et al. This is an open-access article released under the terms of the Creative Commons Attribution (CC BY) license, which allows the unrestricted use, distribution, and reproduction in any medium, provided the original author and source are acknowledged.

Please cite this article as: Joep Schothorst, Griet Van Zeebroeck and Johan M. Thevelein (2017). Identification of Ftr1 and Zrt1 as iron and zinc micronutrient transceptors for activation of the PKA pathway in Saccharomyces cerevisiae. Microbial Cell 4(3): 74-89. doi: 10.15698/mic2017.03.561 
8. Stearman R, Yuan DS, Yamaguchi-Iwai Y, Klausner RD, Dancis A (1996). A permease-oxidase complex involved in high-affinity iron uptake in yeast. Science 271(5255): 1552-1557.

9. Zhao H, Eide D (1996). The yeast ZRT1 gene encodes the zinc transporter protein of a high-affinity uptake system induced by zinc limitation. Proc Natl Acad Sci U S A 93(6): 2454-2458.

10. Seron K, Blondel MO, Haguenauer-Tsapis R, Volland C (1999). Uracil-induced down-regulation of the yeast uracil permease. J Bacteriol 181(6): 1793-1800.

11. Jennings ML, Cui J (2012). Inactivation of Saccharomyces cerevisiae sulfate transporter Sul2p: use it and lose it. Biophys J 102(4): 768-776.

12. Horak J (2003). The role of ubiquitin in down-regulation and intracellular sorting of membrane proteins: insights from yeast. Biochim Biophys Acta 1614(2): 139-155.

13. Lauwers E, Erpapazoglou Z, Haguenauer-Tsapis R, Andre B (2010). The ubiquitin code of yeast permease trafficking. Trends Cell Biol 20(4): 196-204.

14. Felice MR, De Domenico I, Li L, Ward DM, Bartok B, Musci G, Kaplan J (2005). Post-transcriptional regulation of the yeast high affinity iron transport system. J Biol Chem 280(23): 22181-22190.

15. Schepers W, Van Zeebroeck G, Pinkse M, Verhaert P, Thevelein JM (2012). In vivo phosphorylation of Ser21 and Ser83 during nutrientinduced activation of the yeast protein kinase A (PKA) target trehalase. J Biol Chem 287(53): 44130-44142.

16. Thevelein JM, de Winde JH (1999). Novel sensing mechanisms and targets for the cAMP-protein kinase A pathway in the yeast Saccharomyces cerevisiae. Mol Microbiol 33(5): 904-918.

17. Donaton MC, Holsbeeks I, Lagatie O, Van Zeebroeck G, Crauwels M, Winderickx J, Thevelein JM (2003). The Gap1 general amino acid permease acts as an amino acid sensor for activation of protein kinase A targets in the yeast Saccharomyces cerevisiae. Mol Microbiol 50(3): 911-929.

18. Van Zeebroeck G, Rubio-Texeira M, Schothorst J, Thevelein JM (2014). Specific analogues uncouple transport, signalling, oligoubiquitination and endocytosis in the yeast Gap1 amino acid transceptor. Mol Microbiol 93(2): 213-233.

19. Giots F, Donaton MC, Thevelein JM (2003). Inorganic phosphate is sensed by specific phosphate carriers and acts in concert with glucose as a nutrient signal for activation of the protein kinase A pathway in the yeast Saccharomyces cerevisiae. Mol Microbiol 47(4): 1163-1181.

20. Popova Y, Thayumanavan P, Lonati E, Agrochao M, Thevelein JM (2010). Transport and signaling through the phosphate-binding site of the yeast Pho84 phosphate transceptor. Proc Natl Acad Sci U S A 107(7): 2890-2895.

21. Samyn DR, Ruiz-Pavon L, Andersson MR, Popova Y, Thevelein JM, Persson BL (2012). Mutational analysis of putative phosphate- and proton-binding sites in the Saccharomyces cerevisiae Pho84 phosphate: $\mathrm{H}(+)$ transceptor and its effect on signalling to the PKA and PHO pathways. Biochem J 445(3): 413-422.

22. Kankipati HN, Rubio-Texeira M, Castermans D, Diallinas G, Thevelein JM (2015). Sul1 and Sul2 sulfate transceptors signal to protein kinase A upon exit of sulfur starvation. J Biol Chem 290(16): 1043010446.

23. Holsbeeks I, Lagatie O, Van Nuland A, Van de Velde S, Thevelein JM (2004). The eukaryotic plasma membrane as a nutrient-sensing device. Trends Biochem Sci 29(10): 556-564.

24. Schothorst J, Kankipati HN, Conrad M, Samyn DR, Van Zeebroeck G, Popova Y, Rubio-Texeira M, Persson BL, Thevelein JM (2013). Yeast nutrient transceptors provide novel insight in the functionality of membrane transporters. Curr Genet 59(4): 197-206.
25. Thevelein JM, Voordeckers K (2009). Functioning and evolutionary significance of nutrient transceptors. Mol Biol Evol 26(11): 2407-2414.

26. Kriel J, Haesendonckx S, Rubio-Texeira M, Van Zeebroeck G, Thevelein JM (2011). From transporter to transceptor: signaling from transporters provokes re-evaluation of complex trafficking and regulatory controls: endocytic internalization and intracellular trafficking of nutrient transceptors may, at least in part, be governed by their signaling function. Bioessays 33(11): 870-879.

27. Gitan RS, Luo H, Rodgers J, Broderius M, Eide D (1998). Zincinduced inactivation of the yeast ZRT1 zinc transporter occurs through endocytosis and vacuolar degradation. J Biol Chem 273(44): 2861728624.

28. Askwith C, Eide D, Van Ho A, Bernard PS, Li L, Davis-Kaplan S, Sipe DM, Kaplan J (1994). The FET3 gene of $S$. cerevisiae encodes a multicopper oxidase required for ferrous iron uptake. Cell 76(2): 403-410.

29. de Silva DM, Askwith CC, Kaplan J (1996). Molecular mechanisms of iron uptake in eukaryotes. Physiol Rev 76(1): 31-47.

30. Kwok EY, Severance S, Kosman DJ (2006). Evidence for iron channeling in the Fet3p-Ftr1p high-affinity iron uptake complex in the yeast plasma membrane. Biochemistry 45(20): 6317-6327.

31. Cyert MS, Philpott CC (2013). Regulation of cation balance in Saccharomyces cerevisiae. Genetics 193(3): 677-713.

32. Outten CE, Albetel AN (2013). Iron sensing and regulation in Saccharomyces cerevisiae: Ironing out the mechanistic details. Curr Opin Microbiol 16(6): 662-668.

33. Yamaguchi-Iwai Y, Ueta R, Fukunaka A, Sasaki R (2002). Subcellular localization of Aft1 transcription factor responds to iron status in Saccharomyces cerevisiae. J Biol Chem 277(21): 18914-18918.

34. Zhao $H$, Eide $D$ (1996). The ZRT2 gene encodes the low affinity zinc transporter in Saccharomyces cerevisiae. J Biol Chem 271(38): 2320323210.

35. Waters BM, Eide DJ (2002). Combinatorial control of yeast FET4 gene expression by iron, zinc, and oxygen. J Biol Chem 277(37): 33749-33757.

36. Lyons TJ, Gasch AP, Gaither LA, Botstein D, Brown PO, Eide DJ (2000). Genome-wide characterization of the Zap1p zinc-responsive regulon in yeast. Proc Natl Acad Sci U S A 97(14): 7957-7962.

37. Eide DJ (2003). Multiple regulatory mechanisms maintain zinc homeostasis in Saccharomyces cerevisiae. J Nutr 133(5 Suppl 1): 1532S-1535S.

38. Wilson S, Bird AJ (2016). Zinc sensing and regulation in yeast model systems. Arch Biochem Biophys.

39. Gitan RS, Eide DJ (2000). Zinc-regulated ubiquitin conjugation signals endocytosis of the yeast ZRT1 zinc transporter. Biochem J 346 Pt 2(329-336.

40. Znameroski EA, Li X, Tsai JC, Galazka JM, Glass NL, Cate JH (2014). Evidence for transceptor function of cellodextrin transporters in $\mathrm{Neu}$ rospora crassa. J Biol Chem 289(5): 2610-2619.

41. Cai $P$, Wang B, Ji J, Jiang $Y$, Wan L, Tian C, Ma Y (2015). The putative cellodextrin transporter-like protein CLP1 is involved in cellulase induction in Neurospora crassa. J Biol Chem 290(2): 788-796.

42. Schuler D, Wahl R, Wippel K, Vranes M, Munsterkotter M, Sauer N, Kamper J (2015). Hxt1, a monosaccharide transporter and sensor required for virulence of the maize pathogen Ustilago maydis. New Phytol 206(3): 1086-1100.

43. Yendrek CR, Lee YC, Morris V, Liang Y, Pislariu Cl, Burkart G, Meckfessel $M H$, Salehin M, Kessler $H$, Wessler $H$, Lloyd M, Lutton $H$, Teillet A, Sherrier DJ, Journet EP, Harris JM, Dickstein R (2010). A putative 
transporter is essential for integrating nutrient and hormone signaling with lateral root growth and nodule development in Medicago truncatula. Plant J 62(1): 100-112.

44. Bagchi R, Salehin M, Adeyemo OS, Salazar C, Shulaev V, Sherrier DJ, Dickstein R (2012). Functional assessment of the Medicago truncatula NIP/LATD protein demonstrates that it is a high-affinity nitrate transporter. Plant Physiol 160(2): 906-916.

45. Morere-Le Paven MC, Viau L, Hamon A, Vandecasteele C, Pellizzaro A, Bourdin C, Laffont C, Lapied B, Lepetit M, Frugier F, Legros C Limami AM (2011). Characterization of a dual-affinity nitrate transporter MtNRT1.3 in the model legume Medicago truncatula. J Exp Bot 62(15): 5595-5605.

46. Pellizzaro A, Clochard T, Cukier C, Bourdin C, Juchaux M, Montrichard F, Thany S, Raymond V, Planchet E, Limami AM, Morere-Le Paven MC (2014). The nitrate transporter MtNPF6.8 (MtNRT1.3) transports abscisic acid and mediates nitrate regulation of primary root growth in Medicago truncatula. Plant Physiol 166(4): 2152-2165.

47. Gojon A, Krouk G, Perrine-Walker F, Laugier E (2011). Nitrate transceptor(s) in plants. J Exp Bot 62(7): 2299-2308.

48. Ho CH, Frommer WB (2014). Fluorescent sensors for activity and regulation of the nitrate transceptor CHL1/NRT1.1 and oligopeptide transporters. Elife 3(e01917.

49. Ho CH, Lin SH, Hu HC, Tsay YF (2009). CHL1 functions as a nitrate sensor in plants. Cell 138(6): 1184-1194.

50. Walch-Liu P, Forde BG (2008). Nitrate signalling mediated by the NRT1.1 nitrate transporter antagonises L-glutamate-induced changes in root architecture. Plant J 54(5): 820-828.

51. Little DY, Rao H, Oliva S, Daniel-Vedele F, Krapp A, Malamy JE (2005). The putative high-affinity nitrate transporter NRT2.1 represses lateral root initiation in response to nutritional cues. Proc Natl Acad Sci U S A 102(38): 13693-13698.

52. Rogato A, D'Apuzzo E, Chiurazzi M (2010). The multiple plant response to high ammonium conditions: the Lotus japonicus AMT1; 3 protein acts as a putative transceptor. Plant Signal Behav 5(12): 15941596.

53. Lima JE, Kojima S, Takahashi $H$, von Wiren N (2010). Ammonium triggers lateral root branching in Arabidopsis in an AMMONIUM TRANSPORTER1;3-dependent manner. Plant Cell 22(11): 3621-3633.

54. Pastor V, Gamir J, Camanes G, Cerezo M, Sanchez-Bel P, Flors V (2014). Disruption of the ammonium transporter AMT1.1 alters basal defenses generating resistance against Pseudomonas syringae and Plectosphaerella cucumerina. Front Plant Sci 5(231.

55. Zhang B, Pasini R, Dan H, Joshi N, Zhao Y, Leustek T, Zheng ZL (2014). Aberrant gene expression in the Arabidopsis SULTR1;2 mutants suggests a possible regulatory role for this sulfate transporter in response to sulfur nutrient status. Plant J 77(2): 185-197.

56. Zheng ZL, Zhang B, Leustek T (2014). Transceptors at the boundary of nutrient transporters and receptors: a new role for Arabidopsis SULTR1;2 in sulfur sensing. Front Plant Sci 5(710.
57. Rodriguez-Contreras D, Aslan H, Feng X, Tran K, Yates PA, Kamhawi $S$, Landfear SM (2015). Regulation and biological function of a flagellar glucose transporter in Leishmania mexicana: a potential glucose sensor. FASEB J 29(1): 11-24.

58. Stolarczyk E, Le Gall M, Even P, Houllier A, Serradas P, BrotLaroche $E$, Leturque $A$ (2007). Loss of sugar detection by GLUT2 affects glucose homeostasis in mice. PLoS One 2(12): e1288.

59. Stolarczyk E, Guissard C, Michau A, Even PC, Grosfeld A, Serradas $P$, Lorsignol A, Penicaud L, Brot-Laroche E, Leturque A, Le Gall M (2010). Detection of extracellular glucose by GLUT2 contributes to hypothalamic control of food intake. Am J Physiol Endocrinol Metab 298(5): E1078-1087.

60. Thorens B (2015). GLUT2, glucose sensing and glucose homeostasis. Diabetologia 58(2): 221-232.

61. Perez-Torras S, Vidal-Pla A, Cano-Soldado P, Huber-Ruano I, Mazo A, Pastor-Anglada M (2013). Concentrative nucleoside transporter 1 (hCNT1) promotes phenotypic changes relevant to tumor biology in a translocation-independent manner. Cell Death Dis 4(e648.

62. Hundal HS, Taylor PM (2009). Amino acid transceptors: gate keepers of nutrient exchange and regulators of nutrient signaling. Am J Physiol Endocrinol Metab 296(4): E603-613.

63. Hyde R, Cwiklinski EL, MacAulay K, Taylor PM, Hundal HS (2007). Distinct sensor pathways in the hierarchical control of SNAT2, a putative amino acid transceptor, by amino acid availability. J Biol Chem 282(27): 19788-19798.

64. Pinilla J, Aledo JC, Cwiklinski E, Hyde R, Taylor PM, Hundal HS (2011). SNAT2 transceptor signalling via mTOR: a role in cell growth and proliferation? Front Biosci (Elite Ed) 3:1289-1299.

65. Veisova D, Macakova E, Rezabkova L, Sulc M, Vacha $P$, Sychrova $H$, Obsil T, Obsilova V (2012). Role of individual phosphorylation sites for the 14-3-3-protein-dependent activation of yeast neutral trehalase Nth1. Biochem J 443(3): 663-670.

66. App H, Holzer H (1989). Purification and characterization of neutral trehalase from the yeast ABYS1 mutant. J Biol Chem 264(29): 17583-17588.

67. Uno I, Matsumoto K, Adachi K, Ishikawa T (1983). Genetic and biochemical evidence that trehalase is a substrate of cAMPdependent protein kinase in yeast. J Biol Chem 258(18): 10867-10872.

68. Kraakman L, Lemaire $K$, Ma P, Teunissen AW, Donaton MC, Van Dijck P, Winderickx J, de Winde JH, Thevelein JM (1999). A Saccharomyces cerevisiae G-protein coupled receptor, Gpr1, is specifically required for glucose activation of the cAMP pathway during the transition to growth on glucose. Mol Microbiol 32(5): 1002-1012.

69. Van Zeebroeck G, Bonini BM, Versele M, Thevelein JM (2009). Transport and signaling via the amino acid binding site of the yeast Gap1 amino acid transceptor. Nat Chem Biol 5(1): 45-52.

70. Rogers EE, Eide DJ, Guerinot ML (2000). Altered selectivity in an Arabidopsis metal transporter. Proc Natl Acad Sci U S A 97(22): 12356-12360. 